CHERCHER, REPÉRER, AVANCER

\title{
L'AUTONOMIE LOCALE, UN CONCEPT MULTIDIMENSIONNEL : COMMENT LE DÉFINIR, COMMENT LE MESURER ET COMMENT CRÉER UN INDICE D'AUTONOMIE LOCALE COMPARATIF ?
}

Nicolas Keuffer

De Boeck Supérieur | « Revue internationale de politique comparée »

2016/4 Vol. 23 | pages 443 à 490

ISSN 1370-0731

ISBN 9782807390669

Article disponible en ligne à l'adresse :

https://www.cairn.info/revue-internationale-de-politiquecomparee-2016-4-page-443.htm

\section{Pour citer cet article :}

Nicolas Keuffer, «L'autonomie locale, un concept multidimensionnel : comment le définir, comment le mesurer et comment créer un indice d'autonomie locale comparatif ? », Revue internationale de politique comparée 2016/4 (Vol. 23), p. 443-490.

Distribution électronique Cairn.info pour De Boeck Supérieur.

(C) De Boeck Supérieur. Tous droits réservés pour tous pays.

La reproduction ou représentation de cet article, notamment par photocopie, n'est autorisée que dans les limites des conditions générales d'utilisation du site ou, le cas échéant, des conditions générales de la licence souscrite par votre établissement. Toute autre reproduction ou représentation, en tout ou partie, sous quelque forme et de quelque manière que ce soit, est interdite sauf accord préalable et écrit de l'éditeur, en dehors des cas prévus par la législation en vigueur en France. Il est précisé que son stockage dans une base de données est également interdit. 


\section{L'AUTONOMIE LOCALE, UN CONCEPT MULTIDIMENSIONNEL : COMMENT LE DÉFINIR, COMMENT LE MESURER ET COMMENT CRÉER UN INDICE D'AUTONOMIE LOCALE COMPARATIF?}

\section{Nicolas KEUFFER}

L'autonomie locale, caractéristique centrale des gouvernements locaux, a été toujours plus valorisée au cours des dernières décennies ${ }^{1,2}$. En effet, les réformes décentralisatrices, transférant du pouvoir et des responsabilités politiques vers les niveaux gouvernementaux situés au plus près des citoyens, se sont progressivement et silencieusement répandues à travers le monde depuis les années $1980^{3}$. Tant l'autonomie locale que la décentralisation ont été recommandées par de nombreuses institutions européennes et internationales importantes comme des outils clés de la «bonne gouvernance $»^{4}$. Cette dernière sous-tend une forte implication des

1. Une version ultérieure de cet article a été présentée, en anglais, au $24^{\mathrm{e}}$ Congrès mondial de science politique de l'Association Internationale de Science Politique (AISP), qui s'est tenu du 23 au 28 juillet 2016 à Poznań en Pologne.

2. KUHLMANN S., WOLLMANN H., Introduction to Comparative Public Administration. Administrative Systems and Reforms in Europe. Edward Elgar, 2014.

3. IVANYNA M., SHAH A., «How Close Is Your Government to Its People? Worldwide Indicators on Localization and Decentralization », Economics, The Open-Access, Open-Assessment E-Journal, vol. $3, \mathrm{n}^{\circ} 8,2014$, p. 1-62.

4. UNITED CITIES AND LOCAL GOVERNMENTS (UCLG), « Decentralization and local democracy in the world », First global Report by United Cities and Local Governments, U.S.A, 2008 ; UN-HABITAT, «International Guidelines on Decentralisation and Access to Basic Services for all », United Nations Human Settlement Programme, Nairobi, 2009; WHITE S., Government Decentralization in the $21^{\text {st }}$ Century, Washington, Center for Strategic and International Studies, 2011 ; ORGANISATION FOR ECONOMIC CO-OPERATION AND DEVELOPMENT (OECD), « Development Evaluation: Lessons Learned on Donor Support to Decentralisation and Local Governance », DAC Evaluation Series, OECD Publishing, 2004, p. 1-83 ; HUNTER J., SHAH A., « Applying a Simple Measure of Good Governance to the Debate on Fiscal Decentralization », Policy Research Working Paper, n 1894 , 1998, p. 1-35. Dans le cadre de l'expérience opérationnelle de la Banque Mondiale, par exemple, plus d'un quart des opérations de politique de développement approuvées au cours des exercices 1995-2005 répertoriaient au moins une condition contenant la thématique de la décentralisation (KAISER K., « Decentralization Reforms », in COUDOUEL A., PATERNOSTRO S., Analysing the Distributional Impact of Reforms, Washington DC, World Bank, 2006, p. 313-353). 
citoyens dans le processus politique, une plus grande obligation pour les décideurs de rendre des comptes, l'amélioration de l'efficacité économique, l'encouragement de la saine compétition locale et de la coopération interfonctionnelle, le soutien à l'expérimentation dans le cadre de certaines politiques publiques, et la protection de la stabilité macroéconomique et politique ${ }^{5}$. Ainsi, le Conseil de l'Europe a adopté en 1985 la Charte européenne de l'autonomie locale (ci-après: la Charte), un instrument juridique international assurant la protection, l'évaluation et la promotion des principes de décentralisation et d'autonomie locale. Ce dessein ambitieux «suppose l'existence de collectivités locales dotées d'organes de décision démocratiquement constitués et bénéficiant d'une large autonomie quant aux compétences, aux modalités d'exercice de ces dernières et aux moyens nécessaires à l'accomplissement de leur mission ${ }^{6}$.

Un objet récurrent dans les champs d'études consacrés au gouvernement local, au fédéralisme, à la décentralisation et aux phénomènes urbains est la nature des relations entre les gouvernements centraux et locaux et, plus spécifiquement, la mesure dans laquelle ces derniers disposent d'autonomie pour décider de leur système politique, des politiques publiques locales et des biens et services qu'ils offrent. Selon Goldsmith ${ }^{7}$, il est important de se préoccuper de l'autonomie locale, car elle représente la condition nécessaire de la capacité des représentants locaux à répondre aux exigences, besoins et problèmes des citoyens. Dans cette perspective, plus l'autonomie dont ils jouissent est élevée, plus leur responsabilité, leur réactivité et leur obligation de rendre des comptes devraient l'être également. Exprimant la conception libérale millienne classique du gouvernement local, elle peut également accroître la diversité et l'innovation politique. En outre, de par son possible impact sur l'intérêt pour la politique, le développement du leadership et l'éducation démocratique des citoyens à l'échelon local,

5. HANKLA C., «When is Fiscal Decentralization Good for Governance? », Publius: The Journal of Federalism, vol. 39, n 4, 2009, p. 632-650; TREISMAND., The Architecture of Government: Rethinking Political Decentralization, Cambridge, Cambridge University Press, 2007 ; ANDREWS C.W., DE VRIES M.S., «High Expectations, Varying Outcomes: Decentralization and Participation in Brazil, Japan, Russia and Sweden », International Review of Administrative Sciences, vol. 73, n 3, 2007, p. 424-451.

6. CONSEIL DE L'EUROPE, Charte européenne de l'autonomie locale, Strasbourg, CETS n 122 , préambule, 1985a (http ://www.coe.int/fr/web/conventions/full-list/-/conventions/treaty/122, consulté le $31 / 08 / 16)$.

En 2016, 47 États membres du Conseil de l'Europe ont ratifié la Charte européenne de l'autonomie locale. Celle-ci a servi de modèle pour l'élaboration d'un projet de texte d'une charte mondiale de l'autonomie locale par le Centre des Nations Unies pour les établissements humains (CNUEH-Habitat) et la Coordination des associations mondiales des villes et autorités locales (CAMVAL). L'organisation Cités et Gouvernements Locaux Unis (CGLU) a déclaré en 2004 que l'adoption d'une charte mondiale de l'autonomie locale demeurait l'un de ses principaux objectifs.

7. GOLDSMITH M., «Autonomy and City Limits », in JUDGE D., STOCKER G., WOLMAN H., Theories of Urban Politics, London, Sage, 1995, p. 228-252. 
l'autonomie locale peut aussi agir comme un contre-pouvoir vis-à-vis des niveaux gouvernementaux supérieurs. Bien entendu, ces « valeurs de gouvernance » devraient être compensées par les arguments tels que l'intérêt national légitime et l'équité dans les choix publics locaux ${ }^{8}$. Enfin, elle peut permettre la distribution efficiente des services publics, ce qui contribue à légitimer le gouvernement local.

Utilisant le terme «autonomie» pour décrire «la capacité des gouvernements locaux à prendre des décisions à propos des services qu'ils offrent sans interférence du centre », Page ${ }^{9}$ met néanmoins en garde les académiques traitants de ce concept contre sa dimension normative et la variété des vues et significations pouvant être comprises au travers de ce «label». En lien avec cet argument, il se trouve qu'il n'existe pas de définition établie de l'autonomie locale et que la littérature scientifique n'est pas très précise en ce qui concerne l'opérationnalisation de ses diverses composantes ${ }^{10}$. D'autres difficultés ont été signalées en ce qui concerne les études comparatives. Celles-ci sont notamment liées à la terminologie, à la traduction et la contextualité du concept ${ }^{11}$, à l'hétérogénéité des modèles territoriaux, conduisant à diverses notions du «local ${ }^{12}$, au manque de données comparables relatives au niveau local ${ }^{13}$, ou encore à la nécessité de prendre en compte la dimension sectorielle des politiques publiques dans le cadre de l'analyse institutionnaliste ${ }^{14}$.

8. WOLMAN H., «Decentralization: What It Is and Why We Should Care », in BENNET R.J., Decentralization, Local Governments, and Markets: Towards a Post-welfare Agenda, Oxford, Clarendon Press, 1990, p. 32.

9. PAGE E.C., « The Value of Local Autonomy », Local Government Studies, vol. 8, n 4, 1982, p. 21. 10. WOllmanN H., MARCOU G. (eds.), The Provision of Public Services in Europe: Between State, Local Government and Market, Cheltenham, Edward Elgar, 2010 ; VETTER A., Local Politics: A Resource for Democracy in Western Europe? Local Autonomy, Local Integrative Capacity, and Citizens'Attitudes Towards Politics, Lexington Books, 2007 ; HANSEN T., KLAUSEN J.E., « Between the Welfare State and Local government Autonomy », Local government Studies, vol. 28, n 4, 2002, p. 47-66; PIERRE, J., «Assessing Local Autonomy », in KING D.S., PIERRE J., Challenges to Local Government, London, SAGE Publications, 1990, p. 37-54 ; CLARK G.L., «A Theory of Local Autonomy », Annals of the Association of American Geographers, vol. 74, n² 2, 1984, p. 195-208.

11. KUHLMANN S., WOLLMANN H., op. cit., 2014 ; POLLITT C., «Decentralization: A Central Concept in Contemporary Public Management », in FERLIE E., LYNN L.E. Jr., POLLITT C., The Oxford Handbook of Public Management, Oxford, Oxford University Press, 2005, p. 371-397 ; CITÉS ET GOUVERNEMENTS LOCAUX UNIS (CGLU), « Decentralization and Local Democracy in the World », First Global Report by United Cities and Local Governments, U.S.A., 2008.

12. PAGE E.C., GOLDSMITH M., Central and Local Government Relations: A Comparative Analysis of West European Unitary States, London, Sage, 1987, p. 3.

13. BOUCKAERT G., KUHLMANN S., «Conclusion: Tensions, Challenges, and Future 'Flags' of Local Public Sector Reforms and Comparative Research », in KUHLMANN S., BOUCKAERT G., Local Public Sector Reforms in Times of Crisis. National Trajectories and International Comparisons, London, Macmillan, 2016, p. 347-353.

14. WOLMAN H., «Comparing Local Government Systems across Countries: Conceptual and Methodological Challenges to Building a Field of Comparative Local Government Studies », Environment and Planning C: Government and Policy, n 26, 2008, p. 87-103 ; BRAUN D., Public 
L'objectif de cet article est donc de s'appuyer sur les théories de la décentralisation et du gouvernement local, ainsi que sur des études empiriques y relatives, afin de proposer une définition de l'autonomie locale et une opérationnalisation de celle-ci. Cela conduit à l'élaboration d'un « indice d'autonomie locale» (IAL) théoriquement légitimé qui sera systématiquement appliqué à 39 pays européens dans une perspective comparative et en tenant compte de la dimension sectorielle des politiques publiques. La conceptualisation et la mesure de l'autonomie locale sont fondamentales parce qu'elles permettent de tester comparativement si la décentralisation engendre effectivement les implications attendues pour la démocratie locale et pour l'efficacité économique.

Cet article est donc structuré comme suit. Premièrement, le concept d'autonomie locale, ses liens avec celui de décentralisation, ses définitions et approches, ainsi que les études ayant comparativement considéré et mesuré l'autonomie locale, sont présentés pour mettre en exergue les débats existants et les dimensions constitutives prises en compte dans la littérature. Deuxièmement, une définition et une opérationnalisation de l'autonomie locale sont proposées, avant l'IAL et la méthodologie sous-jacente. Troisièmement, les principaux résultats qui en résultent sont présentés. Finalement, l'article s'achève par la discussion de ses principales contributions et pistes d'évolution.

\section{Considérations théoriques}

\section{Le concept d'autonomie locale et ses liens avec celui de décentralisation}

Comme Fleurke et Willemse ${ }^{15}$ l'ont fait remarquer, l'autonomie locale et la décentralisation ont été traitées comme des concepts étroitement associés, l'autonomie locale étant censée donner une valeur positive à la décentralisation ${ }^{16}$, mais leur relation variant en fonction de l'approche de la décentralisation considérée.

\footnotetext{
Policy and Federalism, Aldershot, Ashgate, 2000 ; WOLLMANN H., « Comparing Local Government Reforms in England, Sweden, France and Germany », Wüstenrot Stiftung, 2000, (www.wuestenrotstifung.de/download/local-government, consulté le 31/08/2016) ; MARCOU G., «Les responsabilités des collectivités locales en Europe. Situation en 2007 », Étude du Comité européen sur la démocratie locale et régionale (CDRL), Strasbourg, Conseil de l'Europe, 2010 ; KNOEPFEL P., LARRUE C., VARONE F., HILL M., Public Policy Analysis, Bristol: The Policy Press, 2011.

15. FLEURKE F., WILLEMSE R., «Measuring Local Autonomy: A Decision-making Approach », Local Government Studies, vol. 32, $\mathrm{n}^{\circ}$ 1, 2006, p. 71-87.

16. PAGE E. C., op. cit., 1982 ; MARCOU G., «L'autonomie des collectivités locales en Europe : fondements, formes et limites », Les Entretiens de la Caisse des Dépôts sur le développement local, Paris, Éditions de l'Aube/SECPB, 1999, p. 31-83 ; KING D., STOKER G. (eds.), Rethinking local democracy, Palgrave, 1996.
} 
L'approche la plus intuitive est certainement la décentralisation appréhendée comme un «processus dynamique» de transfert de compétences et de responsabilités dans la gestion de politiques publiques du centre vers les unités infranationales sur un territoire donné ${ }^{17}$. Dans ce cas, les valeurs sous-jacentes de ces réformes décentralisatrices délibérées sont non seulement liées à l'autonomie locale, mais aussi à l'efficience, l'efficacité et la démocratisation du processus politique. À cet égard, trois formes de décentralisation ont été distinguées en administration publique, par rapport à l'ampleur du transfert vers le bas et au niveau d'autonomie accordée à des entités infranationales pour la fourniture d'un service public défini dans un contexte spécifique ${ }^{18}$.

La première est la dévolution, qui se réfère au transfert de certains pouvoirs et ressources vers des autorités autonomes démocratiquement élues (décentralisation politique), situées à des échelons inférieurs et ayant le pouvoir de prélever des impôts et des taxes pour financer les services qu'elles désirent fournir (décentralisation fiscale). La deuxième est la délégation, qui implique le transfert de certaines responsabilités et financements aux autorités inférieures, généralement des unités gouvernementales semiautonomes menant à bien la tâche déléguée (décentralisation administrative). La troisième est la déconcentration, qui suppose que l'autorité supérieure conserve ses pouvoirs et responsabilités pour une certaine fonction alors que son exécution est prise en charge par ses bureaux sur le terrain, qui ne bénéficient d'aucune légitimité démocratique.

Même si ces formes de décentralisation impliquent divers degrés d'autonomie locale, l'ampleur de la décentralisation descendante - ou inversement de la centralisation ascendante - n'est pas équivalente au degré effectif d'autonomie locale, car elle ne prend pas en considération la situation

17. ROLlA G., «Autonomy: a Guiding Criterion for Decentralizing Public Administration », International Review of Administrative Sciences, $\mathrm{n}^{\circ}$ 64, 1998, p. 27-39; REZAZADEH R., "The Concept of Centralization and Decentralization: an Analysis and Evaluation », International Review of Administrative Sciences, $\mathrm{n}^{\circ}$ 27, 1961, p. 425-430 ; BENNET R.J. (eds), Decentralization, Local Governments, and Markets: Towards a Post-Welfare Agenda, Oxford, Clarendon Press, 1990 ; DE VRIES M.S., «The Rise and Fall of Decentralization: A Comparative Analysis of Arguments and Practices in European Countries », European Journal of Political Research, vol. 38, n 2, 2000, p. 193-224 ; FALLETI T.G., «A Sequential Theory of Decentralization: Latin American Cases in Comparative Perspective », The American Political Science Review, vol. 99, n 3, 2005, p. 327-346.

18. BIRD R.M., « Threading the Fiscal Labyrinth: Some Issues in Fiscal Decentralization », National Tax Journal, vol. 46, n² 2, 1993, p. 207-227 ; HUTCHCROFT P.D., « Centralization and Decentralization in Administration and Politics: Assessing Territorial Dimensions of Authority and Power », Governance: An International Journal of Policy and Administration, vol. 14, $\mathrm{n}^{\circ}$ 1, 2001, p. 23-53 ; SCHNEIDER A., «Decentralization: Conceptualization and Measurement», Studies in Comparative International Development, vol. 38, n³ 3, 2003, p. 32-56 ; KUHLMANN S., WAYENBERG E., «Évaluer l'impact institutionnel dans les systèmes à niveaux multiples : conceptualisation des effets de la décentralisation dans une approche comparative », Revue Internationale des Sciences Administratives, vol. 82, $\mathrm{n}^{\circ} 2$, 2016, p. 247-267. 
initiale et le processus à long terme de construction de l'État ${ }^{19}$. Dans les pays en voie de développement, par exemple, le résultat de la décentralisation est l'autonomie locale, qui est le mécanisme par lequel les autres effets attendus de la décentralisation se produisent, alors que dans certains pays fédéraux, les autorités locales autonomes préexistaient le gouvernement central. Cela conduit, d'une part, à la confusion conceptuelle mise en évidence par Elazar : «La non-centralisation n'est pas identique à la décentralisation. [...] La décentralisation implique l'existence d'une autorité centrale, un gouvernement central. Le gouvernement qui peut décentraliser peut re-centraliser s'il le désire. Par conséquent, dans les systèmes décentralisés, la diffusion du pouvoir est en fait une question de grâce, non de droit, et, comme l'histoire le révèle, dans le long terme, elle est généralement traitée comme telle. Dans un système politique non centralisé, le pouvoir est si diffus qu'il ne peut pas légitimement être centralisé ou concentré sans casser la structure et l'esprit de la constitution $»^{20}$. D'autre part, cela implique, pour l'objectivation des variations nationales et/ou diachroniques de l'autonomie locale, de considérer la décentralisation comme un «état statique », l'ampleur de la décentralisation étant ainsi une caractéristique du système politico-administratif ${ }^{21}$. Dans ce cas, les différentes modalités de répartition du pouvoir peuvent être comparées en notant leurs positions relatives le long d'un continuum heuristique centralisation-décentralisation, dont les extrêmes n'existent pas dans la réalité : «la décentralisation totale nécessiterait le dépérissement de l'État alors que la centralisation totale mettrait en péril la capacité de l'État à remplir sa fonction $»^{22}$.

Dans cette perspective "statique », le degré de décentralisation implique plus ou moins d'autonomie dont jouit le gouvernement local. Une telle autonomie locale est traditionnellement envisagée dans sa relation «verticale » à l'État. Elle est en effet mobilisée, selon $\mathrm{Marcou}^{23}$, pour décrire la situation revendiquée par un gouvernement local envers le gouvernement central et, étant mise en place « dans » l'État, elle est en même temps définie «par» l'État. L'article définitionnel et classificatoire bien connu de Clark ${ }^{24}$

19. BENNET R.J., op. cit., 1990.

20. ELAZAR D.J., «Federalism vs. Decentralization: The Drift from Authenticity », Publius, vol. 6, $\mathrm{n}^{\circ} 4,1976$, p. 13.

21. FESLER J.W., «Approaches to the Understanding of Decentralization », The Journal of Politics, vol. 27, n³ 3, 1965, p. 536-566 ; SMITH B.C., Decentralization: The territorial dimension of the state, London, George Allen and Unwin, 1985 ; TREISMAN D., « Defining and Measuring Decentralization: A Global Perspective », 2002, (Unpublished paper, http ://www.sscnet.ucla.edu/polisci/faculty/ treisman/Papers/defin.pdf, consulté le 31/08/16); MUELLER S., Theorising Decentralisation. Comparative Evidence from Sub-national Switzerland. Colchester, ECPR Press, 2015.

22. FESLER J.W., « Centralization and Decentralization », in SILLS D.L., International Encyclopaedia of the Social Sciences, vol. 2, New York: The Macmillan Company and The Free Press, 1968, p. 331.

23. MARCOU G., op. cit., 1999.

24. CLARK G.L., op. cit., 1984. 
a théorisé l'autonomie locale en plaçant précisément les pouvoirs locaux en relation avec les niveaux gouvernementaux supérieurs. Après avoir traduit les deux principes dépendants du pouvoir juridique de Jeremy Bentham ${ }^{25}$ du champ des pouvoirs individuels vers celui des pouvoirs institutionnels, l'auteur définit l'autonomie locale au travers de deux pouvoirs spécifiques : l'initiation et l'immunité. L'initiation est la compétence des autorités locales à effectuer des tâches dans l'intérêt local. En revanche, l'immunité signifie la possibilité pour une autorité locale d'agir sans être sous le contrôle des niveaux gouvernementaux supérieurs. Quant à l'autonomie, elle «définit l'étendue de la discrétion locale en ce qui concerne les fonctions, les actions et le comportement légitime du gouvernement local. [...] La discrétion, ou la capacité des gouvernements locaux à remplir à leur manière leurs propres objectifs conformément à leurs propres normes de mise en œuvre, dépend de la précision préalable de l'autonomie locale ${ }^{26}$. L'approche de Clark pose donc une sorte de séquence de conditions qui, si elles sont satisfaites, impliquent une plus grande autonomie locale : les pouvoirs d'initiation et d'immunité sont légitimes à condition que des droits d'agir de cette manière existent préalablement. L'étendue des deux pouvoirs respectifs indique la configuration de l'autonomie locale et cela détermine à son tour la discrétion locale, à savoir à la fois la liberté de décider de la gamme des fonctions à remplir et la manière de le faire effectivement.

En ce qui concerne la direction de la décentralisation au sein de l'État, Gurr et King ${ }^{27}$ ont tenté d'intégrer une variété de perspectives théoriques (en particulier l'analyse néo-marxiste de la fin des années 1970 et des années 1980) portant sur l'autonomie relative au sein de leur «approche centrée sur l'État ${ }^{28}$. Ils se sont concentrés non pas uniquement sur les limites imposées par des niveaux gouvernementaux supérieurs à un gouvernement local, mais aussi sur une multitude de facteurs locaux : "l'autonomie de l'État local dans les sociétés capitalistes avancées, à n'importe quel moment historique donné, est fonction premièrement de ses relations avec les groupes économiques et sociaux locaux, et deuxièmement de sa relation avec l'État national ou central ${ }^{29}$. Ces deux formes de contrainte sont résumées en deux dimensions qui déterminent ensemble le degré d'autonomie locale. La première dimension, l'autonomie de type I, dépend de facteurs économiques et sociaux à l'échelon local. Par conséquent, la décentralisation est organisée non seulement verticalement, mais aussi «horizontalement» dans l'État. Dans ce cas, elle est concrètement circonscrite par l'ampleur des recettes

25. BENTHAM J., Of Laws in General, London, University of London, The Athlone Press, 1970.

26. CLARK G.L., op. cit., 1984, p. 198-199.

27. GURR T.R., KING D.S., The State and the City, London, Macmillan, 1987.

28. Ibidem, p. 28.

29. Ibidem, p. 56. 
effectives qui peuvent être extraites de l'économie locale, par la capacité des acteurs économiques à contrôler l'agenda politique local et par la présence d'organisations politiques locales et de mouvements sociaux capables de contester ou remodeler les politiques locales mises en œuvre ${ }^{30}$.

Pour assurer sa pérennité, une autorité locale devrait ainsi pouvoir compter sur l'économie locale, ainsi que sur les impôts locaux. Avec le déclin de l'économie locale, un gouvernement local devient de plus en plus dépendant financièrement des échelons gouvernementaux supérieurs. Les contraintes financières de l'autonomie de type I peuvent être surmontées, mais, en retour, les niveaux gouvernementaux supérieurs augmentent leur contrôle sur l'utilisation des ressources financières accordées aux gouvernements locaux. En conséquence, l'autonomie de type II diminue. En effet, cette deuxième dimension concerne la mesure dans laquelle un gouvernement local peut poursuivre ses intérêts sans être limité par des contraintes fixées constitutionnellement, des objectifs stricts accompagnant les subventions, ainsi que des pressions politiques nationales sur les politiques locales ${ }^{31}$. Elle correspond donc en partie au pouvoir d'immunité de Clark $^{32}$, mais comporte aussi des éléments plus détaillés sur les conditions réelles affectant la fourniture locale de biens et services publics. À ce propos, King et Pierre ${ }^{33}$ utilisent, dans le contexte d'une décentralisation bidirectionnelle - soit verticale et horizontale -, les termes « autonomie locale » en référence à l'autonomie de type I et «autonomie du gouvernement local» en référence à l'autonomie de type II : «Le premier signifie l'autonomie de la communauté locale (y compris les gouvernements locaux, les organisations qui participent à des partenariats public-privé, les agences, etc.) et le second fait référence à l'autonomie dont les gouvernements locaux (peuvent) profiter dans le cadre de l'État ${ }^{34}$.

S'agissant de la conceptualisation et de la définition de l'autonomie locale, la Charte, ouverte à la signature par les États membres du Conseil de l'Europe en 1985 en tant que convention juridiquement contraignante, constitue un changement crucial $^{35}$. Après avoir été définie plutôt

30. Ibidem.

31. Ibidem.

32. CLARK G.L., op. cit., 1984.

33. KING D.S., PIERRE J., «Introduction », in KING D.S., PIERRE J., Challenges to Local Government, London, SAGE Publications, 1990, p. 9-12.

34. KING D.S., PIERRE J., op. cit., 1990, cités par FLEURKE F. and WILLEMSE R., « Approaches to Decentralization and Local Autonomy: A Critical Appraisal », Administrative Theory \& Praxis, vol. 26, $\mathrm{n}^{\circ} 4,2004$, p. 536 .

35. Cet article est axé sur l'«autonomie locale» principalement dans le sens traditionnel de l' «autonomie des gouvernements locaux ». Mais nous allons utiliser la première expression, tel que cela a été d'usage dans les champs d'études consacrés au gouvernement local et aux phénomènes urbains, même pour se référer au système de relations intergouvernementales uniquement. En outre, 
négativement/déductivement comme un droit des autorités locales démocratiquement élues à résister aux contraintes imposées, l'autonomie locale a vu son sens s'élargir au fil du temps pour inclure la capacité positive à réaliser les intérêts locaux, ainsi que le moyen de mettre en œuvre d'autres valeurs ${ }^{36}$. Ceci se retrouve également dans la Charte: «Par autonomie locale, on entend le droit et la capacité effective pour les collectivités locales de régler et de gérer, dans le cadre de la loi, sous leur propre responsabilité et au profit de leurs populations, une part importante des affaires publiques ${ }^{37}$. La définition de l'autonomie locale intègre donc la notion de discrétion (« la capacité des gouvernements locaux à remplir à leur manière leurs propres objectifs ») que $\mathrm{Clark}^{38}$ voyait comme sa conséquence logique. Cette définition plus large de l'autonomie locale implique le droit de gérer les affaires publiques dans les limites de la loi, mais aussi les ressources politiques, administratives et financières dont un gouvernement local doit disposer librement afin de le réaliser concrètement. Il convient, certes, de garder à l'esprit que la Charte est une démonstration de la volonté politique de donner du contenu aux principes de la démocratie et des droits de l'homme à tous les niveaux de l'administration territoriale ${ }^{39}$. L'objectif déclaré, « compenser le manque de normes européennes communes pour mesurer et protéger les droits des collectivités locales ${ }^{40}$, est alors en partie inaccessible. En effet, compte tenu de la façon non explicite et flexible dont certaines exigences sont prescrites, il est indéniable que les particularités juridiques et institutionnelles des différents États membres ont été prises en compte pour rencontrer un large consensus ${ }^{41}$. Néanmoins, en raison

l'expression «local self-government », telle qu'employée dans la version anglophone de la Charte, est traduite par « autonomie locale » dans la version francophone. Pour les questions de terminologie, voir KUHLMANN et WOLLMANN (op. cit.) et MARCOU (MARCOU G., « Les collectivités locales dans les constitutions des états unitaires en Europe », Les Nouveaux Cahiers du Conseil constitutionnel, vol. 42, n 1, 2014, p. 63-87. Pour le texte et le tableau des signatures et ratifications du traité, voir Conseil de l'Europe, op. cit., 1985a.

36. CHAPMAN J.L., «Local Government Autonomy and Fiscal Stress: The Case of California Counties », State and Local Government Review, vol. 35, n 1, 2003, p. 15-25.

37. CONSEIL DE L'EUROPE, op. cit., 1985a, art. 3.

38. CLARK, G.L., op. cit., 1984, p. 199.

39. CONSEIL DE L'EUROPE. Rapport explicatif de la Charte européenne de l'autonomie locale, 1985b, Strasbourg.

40. Ibidem, p. 2.

41. Selon le rapport explicatif de la Charte européenne de l'autonomie locale (Ibidem), la formulation de certains articles représente indéniablement un compromis politique : le principe de l'autonomie locale n'est pas consacré dans les constitutions de tous les pays membres (art. 2); la part des responsabilités locales, à laquelle la séquence « une part importante des affaires publiques » (art. 3) fait référence, n'est pas quantifiée, et des expressions telles que « les affaires locales » et « propres affaires » ont été rejetées, car les affaires étant sous la responsabilité des autorités locales diffèrent grandement selon les traditions des États membres; la portée des responsabilités qui devraient appartenir aux collectivités locales européennes n'est pas précisée, tout comme la portée de la consultation (art. 4); en ce qui concerne la protection des frontières des collectivités locales, il a été considéré comme irréaliste d'attendre de la 
de l'intégration simultanée des doubles critères de droit et de capacité effective, ainsi que le grand nombre de principes substantiels sur lesquels doit se fonder l'autonomie locale, la Charte constitue un grand pas en avant vers l'objectivation de l'autonomie locale.

\section{Les différents débats et approches autour des multiples dimensions de l'autonomie locale}

En tant qu'expression des modalités relationnelles (verticales) entre niveaux de gouvernement ${ }^{42}$, nous affirmons qu'il n'y a pas « une » théorie de l'autonomie locale, mais plutôt divers débats, approches et définitions entourant les différentes dimensions de l'autonomie locale. En premier lieu, cela se rapporte au fait que le concept d'autonomie locale est trop diversifié pour qu'une seule théorie existe ${ }^{43}$. En second lieu, cela vient de la nature interdisciplinaire du concept d'autonomie locale, entendu à la fois comme droit et capacité effective : droit, administration publique, économie

communauté - entendu au sens de population - locale d'avoir le pouvoir de veto sur ces changements (art. 5) ; même si les ressources financières sont considérées comme la condition nécessaire pour une autonomie locale significative, la proportion des revenus qui découle des impôts et taxes locaux (« une partie au moins », art. 9, al. 3), ainsi que la proportion de subventions globales comparées aux subventions affectées à des projets spécifiques ( « autant que possible », art. 9, al. 7) ne sont pas prescrites précisément ; un recours à un remède extraordinaire a été intégré comme voie de recours juridictionnel (art. 11) ; enfin, la possibilité a été donnée aux parties d'exclure certaines dispositions (le système de «noyau obligatoire », art. 12). Plutôt qu'à un corpus de dispositions juridiquement contraignantes, la Charte s'apparente donc davantage à un instrument conventionnel menant progressivement à une harmonisation volontaire des systèmes juridiques des États-membres.

42. Bien que cet article soit axé sur l'autonomie locale en tant que concept relatif, il est important de mentionner brièvement que l'autonomie locale est également traitée dans la littérature, comme PRATCHETT (PRATCHETT L., «Local Autonomy, Local Democracy and the 'New Localism' », Political Studies, vol. 52, 2004, p. 358-375) le suggère, en se concentrant sur ses impacts sur le bien-être des résidents (WOLMAN H., GOLDSMITH M., « Local Autonomy as a Meaningful Analytic Concept », Urban Affairs Quartely, vol. 26, n 1, 1990, p. 3-27 ; WOLMAN H., GOLDSMITH M., Urban Politics and Policy, Oxford, Basil Blackwell, 1992), ainsi que sur la façon dont elle est subjectivement assimilée par les citoyens, élus ou bureaucrates locaux (LADNER A., «Finanzkompetenz in der Gemeinde - Ein Überblick über die Praxis », in ENG F., GLATTHARD A., KÖNIG B.H., Finanzen der öffentlichen Hand Nr. 5, Finanzföderalismus, Bern, Emissionszentrale der Schweizer Gemeinden, 1994, p. 65-85,). Cette dernière approche, basée sur la pensée tocquevillienne, souligne les valeurs de participation, d'engagement, d'indépendance et d'attachement émotionnel, et comprend l'autonomie locale comme la capacité des gouvernements locaux à définir et à développer, au travers de l'activité politique, leurs sens du lieu et identité propres. L'autonomie locale est ainsi considérée non pas comme quelque chose de statique, accordé ou possédé par un gouvernement local, mais plutôt comme une construction relationnelle complexe (DE FILIPPIS J., « Alternatives to the 'New Urban Politics': Finding Locality and Autonomy in Local Economic Development », Political Geography, n 18, 1999, p. 973-990) se matérialisant, d'un point de vue local, par la recherche constante de stratégies locales de résistance et de contestation dans le cadre des relations de pouvoir (BROWN M.P., « The Possibility of Local Autonomy », Urban Geography, n 13, 1992, p. 257-279).

43. KJELLBERG F., « The Changing Values of Local Government », Annals of the American Academy of Political and Social Science, $\mathrm{n}^{\circ}$ 540, 1995, p. 40-50. 
et science politique, toutes mettent en évidence une dimension spécifique de celui-ci ${ }^{44}$. Cette section vise donc à présenter les différentes approches de l'autonomie locale.

\section{L'approche légaliste}

Comme mentionné plus haut, la littérature sur l'autonomie locale est principalement axée sur les réglementations juridiques supra-locales définissant l'autonomie locale de manière défensive, selon un cadre juridique formel. Cette perspective légaliste, principalement investie par des juristes, désigne plusieurs indices juridiques différents.

Le premier correspond aux droits antérieurs - identifiés par Clark ${ }^{45}$ nécessaires à ce que le pouvoir puisse être formellement détenu par les gouvernements locaux, à savoir le droit d'exister et le droit de se prononcer sur les frontières de son territoire. À cet égard, la Charte prescrit qu'une fusion décidée « par le haut » soit interdite puisque « les collectivités locales concernées doivent être consultées préalablement » (art. 5). La question de savoir si ce sont les autorités représentatives qui doivent être consultées, et par quels moyens, n'est néanmoins pas claire et laissée à la discrétion des États signataires. Ces droits antérieurs sont étroitement liés à la situation juridique des gouvernements locaux au sein de la structure institutionnelle étatique qui, selon la Charte (art.2), devrait autant que possible être formalisée constitutionnellement.

Le second indice juridique fait référence à la répartition formelle et générale des compétences entre les différents niveaux gouvernementaux prévue par le cadre juridique. On distingue à ce propos les pays où les gouvernements locaux disposent d'une «compétence générale » à entreprendre des fonctions, et les autres, où les autorités locales doivent trouver une base légale spécifique pour leur action, comme c'est le cas dans la doctrine de l'ultra vires au Royaume-Uni par exemple. Néanmoins, « il est douteux que l'existence du principe de compétence générale implique per se nécessairement un plus grand degré d'autonomie ${ }^{46}$. En effet, cette question de la répartition formelle des compétences est inextricablement liée à celle de la subsidiarité, principe général d'organisation institutionnelle ${ }^{47}$.

44. SCHNEIDER A., op. cit., 2003.

45. CLARK G.L., op. cit., 1984.

46. BLAIR P., « Trends in Local Autonomy and Democracy : Reflections from a European Perspective », in BATLEY R., STOKER G. (eds.), Local Government in Europe. Trends and Developments, London, MacMillan, 1991, p. 51.

47. KEUNEN S., «Local Autonomy and Subsidiarity: A Two-way Principle », Paper presented at the IPSA 24th World Congress of Political Science, Poznan, Poland, 23-28 July 2016. 
La Charte ne mentionne pas explicitement le principe de subsidiarité, mais une définition implicite peut être trouvée à l'art. 4 , intitulé «portée de l'autonomie locale»: «l'exercice des responsabilités publiques doit, de façon générale, incomber, de préférence, aux autorités les plus proches des citoyens. L'attribution d'une responsabilité à une autre autorité doit tenir compte de l'ampleur et de la nature de la tâche et des exigences d'efficacité et d'économie » (al. 3). En plus du choix politique consistant à favoriser les autorités les plus proches de leurs électeurs, quatre critères pour la division du pouvoir entre les autorités sont donc prévus : la nature et l'ampleur de la tâche (deux critères objectifs), ainsi que les deux notions plus subjectives d'efficacité et d'économie ${ }^{48}$. En outre, la Charte prescrit l'obligation pour l'autorité supérieure d'aider l'autorité inférieure dans l'accomplissement de ses tâches lorsque cette dernière ne jouit pas de la capacité suffisante pour rendre l'autonomie locale effective ${ }^{49}$. Enfin, selon l'art. 4, al. 2, « les collectivités locales ont, dans le cadre de la loi, toute latitude pour exercer leur initiative pour toute question qui n'est pas exclue de leur compétence ou attribuée à une autre autorité ». Par conséquent, cela suppose, d'une part, un pouvoir inhérent des autorités locales, suggérant que la subsidiarité est le principe sous-jacent du processus ascendant de construction étatique de la «non-centralisation», et qu'elle est alors seulement une modalité recommandée d'organisation des institutions étatiques en cas de «décentralisation »-pour reprendre la distinction d'Elazar mentionnée précédemment. D'autre part, cela montre que l'attribution officielle des pouvoirs peut interférer avec d'autres mesures juridiques, qui peuvent impliquer des compétences soit de prise de décision soit de contrôle pour la fourniture d'un service public spécifique ${ }^{50}$.

Troisièmement, l'éventail de la supervision formelle peut être utilisé, entre autres, comme un aspect juridique. Considérant les constitutions nationales et européennes, $\mathrm{Marcou}^{51}$, par exemple, définit l'autonomie locale à travers quatre éléments : le droit reconnu à une collectivité territoriale de s'administrer elle-même, le caractère démocratique de cette administration, la vocation générale de sa compétence, et le contrôle de l'État.

Quatrièmement, à l'inverse, l'existence formelle de moyens constitutionnels ou légaux pour protéger l'autonomie locale contre la violation des principes de droit d'existence, de décision sur les limites du territoire, et de compétence pour le libre exercice des responsabilités dans la provision des tâches publiques est parfois considérée comme un indice juridique.

48. CONSEIL DE L'EUROPE, Définition et limites du principe de subsidiarité, Rapport préparé pour le Comité directeur des autorités locales et régionales (CDLR), 1994.

49. Ibidem.

50. WOLMAN H., op. cit., 1990, p. 1991.

51. MARCOU G., op. cit., 1999, p. 34. 


\section{L'approche fonctionnelle}

En ce qui concerne la structure verticale du secteur public, l'attribution des fonctions et des instruments fiscaux aux niveaux gouvernementaux appropriés est un enjeu de longue date dans le cadre de la théorie de la décentralisation fiscale ${ }^{52}$. Les économistes de la première génération de la décentralisation fiscale ont proposé un cadre normatif faisant correspondre l'efficience du système avec un modèle de répartition des tâches basé sur une séparation claire des tâches par niveau (modèle dual) et une forte décentralisation ${ }^{53}$. Dans cette perspective, un gouvernement local autonome peut améliorer son efficacité dans l'accomplissement de ses responsabilités en matière de services publics en les adaptant aux préférences des citoyens (output). Par conséquent, étant donné que l'étendue des fonctions pour lesquelles il est effectivement responsable est liée au degré de décentralisation politique, c'est une indication de l'autonomie locale.

Toutefois, cette indication peut soulever certaines questions ${ }^{54}$. Premièrement, l'importance d'une tâche publique n'est pas la même d'un pays à l'autre. Deuxièmement, le rôle réel des gouvernements locaux peut être flou dans des "modèles administratifs intégrés ${ }^{55}$, où de nombreuses tâches sont partagées entre les niveaux. Troisièmement, même si les compétences ont été attribuées de jure, il est possible que le gouvernement local agisse de facto au nom des gouvernementaux supérieurs (décentralisation administrative), sous leur supervision, et sans réelle discrétion dans la prise de décision (tâches « obligatoires » ou «autorisées $»)^{56}$. Ce sont les raisons pour lesquelles la part des dépenses infranationales directes par rapport aux dépenses publiques totales est loin d'être une mesure précise de l'étendue de ses responsabilités réelles - bien que souvent utilisée en raison du manque de bonnes

52. OATES W.E., Decentralization of the Public Sector, in BENNET R.J., Decentralization, Local Governments, and Markets: Towards a Post-welfare Agenda, Oxford, Clarendon Press, 1990, p. 43-58. 53. BUCHANAN J.M., «Federalism and Fiscal Equity », The American Economic Review, vol. 40, n 4, 1950, p. 583-599; TIEBOUT C.M., «A Pure Theory of Local Expenditures », The Journal of Political Economy, vol. 64, n 5, 1956, p. 416-424 ; MUSGRAVE R.A., The Theory of Public Finance : A Study in Public Economy, New York, McGraw-Hill, 1959; OLSON M., « The Principle of 'Fiscal Equivalence': The Division of Responsibilities among Different Levels of Government », The American Economic Review, vol. 59, n² 2, 1969, p. 479-487; TULLOCK G., « Social Cost and Government Action », The American Economic Review, vol. 59, n², 1969, p. 189-197; OATES W.E., Fiscal Federalism, New York, Harcourt Brace Jovanovich, 1972.

54. BLAIR P., op. cit., 1991, p. 41-58.

55. KUHLMANN, S. and H. WOLLMANN, op. cit., 2014, p. 23.

56. PAGE E.C., Localism and Centralism in Europe: The political and Legal Bases of Local Self-government, Oxford, Oxford University Press, 1991, p. 24 et ss. 
données générales ${ }^{57}$. Les mêmes limites peuvent être adressées à l'autre mesure la plus largement utilisée dans les études sur la décentralisation fiscale, à savoir la part des recettes publiques infranationales par rapport aux recettes totales ${ }^{58}$.

La structure verticale des recettes (input) est de première importance pour l'autonomie locale, dansl'hypothèse - qui ne prend pas en compte le caractère multidimensionnel de l'autonomie financière ${ }^{59}$ - que plus la proportion du revenu du gouvernement local provenant des niveaux gouvernementaux d'ordre supérieur est grande, moins le degré d'autonomie locale est élevé. En outre, en conformité avec la prise en compte dans la Charte du critère de capacité effective, les économistes n'ont pas été les seuls à souligner l'importance des ressources - principalement financières ${ }^{60}$ - pour que les autorités locales soient véritablement autonomes ${ }^{61}$. Ainsi, Vetter définit l'autonomie locale comme « la gamme des fonctions délivrées au niveau local dans un pays et la liberté des autorités locales de prendre des décisions sur la façon de fournir leurs services - la portée de leur discrétion ${ }^{62}$ et construit finalement un indice d'autonomie avec la moyenne des indices des dépenses

57. VAN DE WALlE S., BAKER K., SKELCHER C., «Citizen Support for Increasing the Responsibilities of Local Government in European Countries : A Comparative Analysis », Working paper, 2009, (https://www.researchgate.net/publication/228157459, consulté le 31/08/2016); BELL M.E., EBEL R.D., KAISER K., ROJCHAICHAINTHORN J., Measuring Fiscal Decentralization : A New Perspective. Draft for discussion, The World Bank, 2006 ; SHARPE L.J., « The Growth and Decentralisation of the Modern Democratic State », European Journal of Political Research, vol. $16, \mathrm{n}^{\circ} 3,1988$, p. 365-380.

58. AKAI N., « The Role of Decentralisation Indicators in Empirical Research », in KIM J., LOTZ J., BLÖCHLIGER H., Measuring Fiscal Decentralisation: Concepts and Policies, OECD and Korea Institute of Public Finance, OECD Fiscal Federalism Studies, OECD Publishing, 2013, p. 61-70 ; KIM J., « Measurement of Decentralisation : How Should We Categorise Tax Sharing? », in KIM J., LOTZ J., BLÖCHLIGER H., Measuring Fiscal Decentralisation. Concepts and Policies, OECD and Korea Institute of Public Finance, OECD Fiscal Federalism Studies, OECD Publishing, 2013, p. 47-60. 59. ORGANISATION FOR ECONOMIC CO-OPERATION AND DEVELOPMENT (OCDE), KOREAN INSTITUTE OF PUBLIC FINANCE (KIPF), «Fiscal Federalism 2016: Making Decentralisation Work », OECD Publishing, Paris, 2016.

60. Selon la Charte, l'autonomie financière doit être suffisante et dépendre d'une variété de sources différentes : le «droit, dans le cadre de la politique économique nationale, à des ressources propres suffisantes dont elles peuvent disposer librement» (art. 9, al. 1) ; «proportionnées aux compétences prévues par la Constitution ou la loi » (art. 9, al. 2); des ressources financières composées pour « une partie au moins [...] de redevances et d'impôts locaux dont elles ont le pouvoir de fixer le taux » (art. 9, al. 3) ; «de nature suffisamment diversifiée et évolutive pour leur permettre de suivre, autant que possible dans la pratique, l'évolution réelle des coûts de l'exercice de leurs compétences » (art. 9, al. 4) ; « les procédures de péréquation financière ou des mesures équivalentes [...] ne doivent pas réduire la liberté d'option des collectivités locales dans leur propre domaine de responsabilité » (art. 9, al. 5) ; « les subventions accordées aux collectivités locales ne doivent pas être destinées au financement de projets spécifiques » (art. 9, al. 7) ; « les collectivités locales doivent avoir accès, conformément à la loi, au marché national des capitaux » (art. 9, al. 8).

61. VETTER A., op. cit., 2007 ; KING D.S., PIERRE J., op. cit., 1990 ; PRATCHETT L., op. cit., 2004.

62. VETTER A., op. cit., 2007, p. 99. 
et des recettes locales. En qui concerne les recettes, les ressources les plus importantes d'un gouvernement infranational sont les recettes fiscales et les subventions intergouvernementales ${ }^{63}$. Pour Oates ${ }^{64}$, l'autonomie locale devrait ainsi impliquer en premier lieu pour le gouvernement local le pouvoir sur son propre impôt et sur les instruments de taxation. Comme les normes juridiques et leurs implications ne sont généralement pas directement prises en compte dans les flux financiers ${ }^{65}$, de récents rapports de l'Organisation de Coopération et de Développement Économiques (OCDE) ont essayé de préciser la déclinaison du pouvoir de taxation du gouvernement local comme suit (du plus au moins important) : le pouvoir complet sur les taux et les bases d'imposition, le pouvoir sur les taux d'imposition, le pouvoir sur les bases d'imposition, des ententes de partage fiscal et aucun pouvoir sur les taux et les bases d'imposition ${ }^{66}$.

En ce qui concerne les subventions intergouvernementales, les principales fonctions relevées par la littérature sont l'internalisation des effets de débordement, l'égalisation du déséquilibre fiscal (qui peut également être atteint par un système de péréquation horizontale) et l'amélioration du système fiscal global. Elles peuvent être soit inconditionnelles, si elles désignent des transferts non affectés, soit conditionnelles, lorsqu'elles sont transférées aux gouvernements locaux pour des programmes spécifiques et dont l'utilisation peut ainsi être soumise à une surveillance stricte ${ }^{67}$. En conséquence, l'octroi d'un financement par les niveaux gouvernementaux supérieurs ne réduit pas nécessairement l'autonomie locale ${ }^{68}$. Cependant, il est crucial, selon Oates, que les gouvernements locaux « lèvent une partie importante de leurs fonds propres ${ }^{69}$ afin de préserver leur indépendance sur les dépenses et les incitations existantes et que des décisions fiscales prudentes soient prises localement.

Enfin, la possibilité pour un gouvernement local d'emprunter, afin de disposer de ressources financières supplémentaires dont il peut disposer

63. BERGVALL D., CHARBIT C., KRAAN D.-J., MERK O., «Intergovernmental Transfers and Decentralised Public Spending », OECD Journal on Budgeting, vol. 5, n 4, 2006, p. 112-158.

64. OATES W.E., Fiscal Federalism, Edward Elgar Publishing Limited, Cheltenham, 2011.

65. STEGARESCU D., «Public Sector Decentralization: Measurement Concepts and Recent International Trends », Fiscal Studies, vol. 26, n 3, 2005, p. 301-333.

66. BLÖCHLIGER H., « Measuring Decentralisation: The OECD Fiscal Decentralisation Database », in KIM J., LOTZ J., BLÖCHLIGER H., Measuring Fiscal Decentralisation. Concepts and Policies, OECD and Korea Institute of Public Finance, OECD Fiscal Federalism Studies, OECD Publishing, 2013, p. 15-35 ; BLÖCHLIGER H., KING D., « Less than You Thought : the Fiscal Autonomy of Subcentral Governments ». OECD Economic Studies, 2006/2, OECD Publishing.

67. OATES W. E., «An Essay on Fiscal Federalism ». Journal of Economic Literature, vol. 37, n 3 , 1999, p. 1120-1149 ; BLÖCHLIGER H., op. cit., 2013.

68. DAVEY K. J., «Local Autonomy and Independent Revenues », Journal of Public Administration, vol. 49, 1971, p. 45-50.

69. OATES W.E., op. cit., 1990, p. 50. 
librement, peut également déterminer, dans une certaine mesure, le degré d'autonomie locale ${ }^{70}$.

\section{L'approche organisationnelle}

Cette approche politico-administrative est principalement axée sur la façon dont un gouvernement local s'organise lui-même (auto-gouvernance) pour remplir ses fonctions. Le concept clé dans cette perspective est ainsi la capacité du gouvernement local, qui, parmi les nombreuses définitions existantes, peut simplement être définie comme « la capacité du gouvernement local à exercer ses fonctions de manière efficace et efficiente ${ }^{71}$. Afin d'expliquer la capacité variable des gouvernements locaux dans le monde entier, les auteurs, tout en reconnaissant l'énorme variation existant dans la littérature, distinguent quatre conditions. Premièrement, les « conditions contextuelles » se réfèrent à des déterminants juridiques, socio-économiques et historiques très généraux. Deuxièmement, les « conditions structurelles » ont à voir avec la position relative des gouvernements locaux en termes de responsabilités fonctionnelles et d'autonomie financière. Ce facteur englobe donc les préoccupations de la théorie de la décentralisation fiscale. Troisièmement, les « conditions institutionnelles » sont liées à la taille du gouvernement local, son organisation interne, son budget financier et ses infrastructures. Enfin, les « conditions relatives aux ressources humaines » se réfèrent essentiellement aux questions liées à la gestion du personnel ${ }^{72}$.

Ces conditions sont liées à une préoccupation substantielle, la « capacité du système » à répondre aux préférences collectives des citoyens, et à une préoccupation procédurale, l' « efficacité citoyenne » à contrôler pleinement les décisions du politique en agissant de manière responsable et compétente ${ }^{73}$. En ce sens, l'autonomie locale peut être considérée comme l'élément constitutif « des deux faces de l'autodétermination démocratique ${ }^{74}$. Du point de vue de l'output, elle légitime des choix politiques qui répondent efficacement aux exigences et préférences des citoyens (gouvernement pour le peuple). Du point de vue de l'input, elle légitime les décisions politiques

\footnotetext{
70. RODDEN J., «The Dilemma of Fiscal Federalism: Grants and Fiscal Performance around the World », American Journal of Political Science, vol. 46, n 3, 2002, p. 670-687 ; SWIANIEWICZ P., Local Government Borrowing : Risks and Rewards, OSI/LGI, Budapest, 2004.

71. REDDY P., NEMEC J., DE VRIES M., « The State of Local Government », Public Policy and Administration, vol. 14, $\mathrm{n}^{\circ} 3,2015$, p. 161.

72. REDDY P., NEMEC J., DE VRIES M., op. cit., 2015, p. 162.

73. DAHL R.A., TUFTE E.R., Size and Democracy, London, Oxford University Press, 1973, p. 20.

74. SCHARPF F.W., « Economic Integration, Democracy and the Welfare State », Journal of European Public Policy, vol. 4, 1997, p. 19.
} 
qui ont été déterminées grâce à toute une série de procédures, telles que le débat public, les votes ou les élections (gouvernement par le peuple) ${ }^{75}$.

La dimension démocratique est donc le prérequis nécessaire à la décentralisation politique - ou à la «vraie » municipalisation ${ }^{76}$-, ainsi que l'essence même de l'autonomie locale, car elle implique la création et l'entretien des arènes politiques ${ }^{77}$. En fonction de l'étendue de l'autonomie locale, l'activité politique sous-jacente peut conduire à des profils politiques variés. Premièrement, elle peut contribuer au développement d'une identité locale plus ou moins forte. Deuxièmement, elle peut différemment affecter des éléments relatifs à la structure de la démocratie locale, tels que le type de corps législatif (représentatif ou démocratie directe), l'existence d'autres moyens pour la participation citoyenne au niveau local, le type de suffrage électoral, ou la possibilité de disposer d'organes exécutifs responsables ${ }^{78}$. Troisièmement, elle peut être liée à la marge de manœuvre pour déterminer des éléments centraux du système politique (système électoral majoritaire ou proportionnel, nombre de sièges, circonscriptions électorales, etc.) ${ }^{79}$. Quatrièmement, elle peut avoir un impact sur le statut des élus locaux et les conditions d'exercice de leurs fonctions, selon l'art. 7 de la Charte, ainsi que sur la structure politico-administrative de direction, qui renvoie aux différences entre les systèmes moniste et dual ${ }^{80}$, et sur le rôle des dirigeants politiques locaux ${ }^{81}$. Certains de ces aspects peuvent également avoir un impact sur la possibilité des acteurs locaux à accéder aux niveaux gouvernementaux supérieurs (voir la prochaine sous-section).

L'ampleur de l'autonomie locale détermine le niveau de capacité administrative d'un gouvernement local, considéré comme une organisation fournissant des services publics à l'échelon local ${ }^{82}$, dans l'exécution de fonctions (propres ou déléguées). À cet égard, de nombreux

75. SCHARPF F.W., Governing in Europe: effective and democratic?, Oxford, University Press, Oxford, 1999 ; KERSTING N., VETTER A., Reforming Local Government in Europe: Closing the Gap between Democracy and Efficiency, Opladen, Leske + Budrich, 2003.

76. KUHLMANN S., WOLLMANN H., op. cit., 2014, p. 120.

77. RIKER W., «Six Books in Search of a Subject or Does Federalism Exist and Does it Matter », Comparative Politics, vol. 2, $\mathrm{n}^{\circ} 1,1969$, p. 135-46.

78. CONSEIL DE L'EUROPE, op. cit., 1985a, art. 3, al. 2.

79. EVANS M., «Democracy, Legitimacy and Local Government Electoral Reform », in Local Government Studies, vol. 40, n 1, 2014, p 41-63 ; KUHLMANN S., WAYENBERG E., op. cit., 2016. 80. WOLLMANN H., «Local Government Reforms in Great Britain, Sweden, Germany and France. Between Multi-function and Single-purpose Organisation », Local Government Studies, vol. 30, ${ }^{\circ} 4$, 2004, p. 639-665.

81. HAUS M., SWEETING D., «Local Democracy and Political Leadership : Drawing a Map », Political Studies, vol. 54, 2006, p. 267-288; GOLDSMITH M., LARSEN H., « Local Political Leadership: Nordic Style », International Journal of Urban and Reginal Research, vol. 28, $\mathrm{n}^{\circ} 1$, 2004, p. 121-133 ; MOURITZEN P-E.M., SVARA J., Leadership at the Apex, Pittsburgh, Pittsburgh University Press, 2002.

82. POLLITT C., op. cit., 2005. 
gouvernements locaux européens, confrontés à une complexité croissante des tâches couplée à une diminution des ressources à leur disposition pour les fournir, ainsi qu'aux tendances à la mondialisation et à l'augmentation du niveau d'exigence citoyenne, ont lancé des réformes depuis les années $1980^{83}$. L'autonomie locale peut être, de ce point de vue, considérée comme une ressource institutionnelle nécessaire aux gouvernements locaux pour prendre l'initiative de réformer ${ }^{84}$. Les réformes effectuées ont été influencées par les directives issues des théories économiques de l'organisation, du managérialisme et du néolibéralisme - en particulier le courant connu sous l'expression de Nouvelle Gestion Publique (NGP) - afin d'améliorer sensiblement l'efficience et l'efficacité de la fourniture de services publics ${ }^{85}$. Le mouvement réformateur a visé (de façon non homogène) à redéfinir le rôle et l'action du gouvernement et à renforcer les mécanismes de marché.

$\mathrm{Au}$ niveau local, cette décentralisation horizontale ${ }^{86}$ a mené à une évolution du «gouvernement local» vers la «gouvernance locale ${ }^{87}$, qui dénote la transition vers des réseaux d' « organisations à but unique » ( $v s$ organisations multifonctions) opérant généralement en dehors de l'influence directe des gouvernements locaux ${ }^{88}$. Par ailleurs, les réformes ont également visé au développement des structures internes, des instruments de gestion et des profils du personnel administratif, afin d'augmenter la capacité administrative qu'un gouvernement local a à sa disposition, ce qui «se reflète par l'administration efficace des infrastructures locales et une fourniture locale de biens et services qui a fait ses preuves $»^{89}$.

Ces différents moyens administratifs font partie des leviers concrets soulignés par la Charte pour que l'autonomie locale soit effective : «les collectivités locales doivent pouvoir définir elles-mêmes les structures

83. KERSTING N., VETTER A., op. cit., 2003.

84. KEUFFER N., «Is the Propensity of a Local Government to Reform Dependent on its Local Autonomy? Evidence from Switzerland», Paper presented at the IRSPM Conference 2015, Panel C101 - Special Interest Group in Local Governance, Birmingham, 2015.

85. JOHN P., Local Governance in Western Europe, London, Sage, 2001.

86. BENNET R.J., op. cit., 1990.

87. DENTERS B., ROSE L.E. (eds.), Comparing Local Governance: Trends and Developments. Basingstoke, Palgrave Macmillan, 2005 ; STOKER G., The Comparative Study of Local Governance: towards a Global Approach, in Symposium on Chinese Social Capacity Building in International and Comparative Perspective, Zhejiang University, Hangzhou, China, 2010 ; RHODES R.A.W., « The New Governance: Governing without Government », Political Studies, vol. XLIV, 1996, p. 652-667.

88. WOLLMANN H., MARCOU G., op. cit., 2010 ; LOUGHLIN J. « 'The Hybrid State' : Reconfiguring Territorial Governance in Western Europe », in Perspectives on European Politics and Society, vol. 10, $\mathrm{n}^{\circ} 1$, 2009, p. 51-68 ; PETERS B.G., PIERRE J., «Governance without Government? Rethinking Public Administration », Journal of public administration research and theory, vol. 8, $\mathrm{n}^{\circ} 2$, 1998, p. 223-243.

89. REDDY P., NEMEC J., DE VRIES M., op. cit., 2015, p. 165. 
administratives internes dont elles entendent se doter » (art. 6, al. 1) ; «Le statut du personnel des collectivités locales doit permettre un recrutement de qualité, fondé sur les principes du mérite et de la compétence ; à cette fin, il doit réunir des conditions adéquates de formation, de rémunération et de perspectives de carrière » (art. 6, al. 2). À ce propos, les mécanismes internes, comme le budget local par exemple, peuvent être considérés comme des indications partielles de l'ampleur de l'autonomie locale, tout comme la proportion de main d'œuvre ou des salaires du personnel local. Mais ces deux dernières mesures rencontrent les mêmes limites que celle de la proportion des dépenses locales (voir la précédente sous-section).

\section{L'approche politique des relations intergouvernementales}

L'intérêt de cette approche n'est pas porté sur une étude macro des relations intergouvernementales en tant que relations financières, organisationnelles, juridiques et politiques qui existent entre les niveaux constitutifs d'un État, mais sur les « relations verticales bilatérales qui s'opèrent effectivement dans le cadre du politique, dans le sens des dimensions relatives aux acteurs et aux processus de la vie politique $\gg{ }^{90}$. Celles-ci placent dans un jeu de pouvoir intergouvernemental des individus organisés en partis politiques, en associations, en groupes de pression ou en mouvements sociaux. Hutchcroft ${ }^{91}$ tente d'expliquer et de relier les points de vue administratif et politique sur la centralisation et la décentralisation afin de décrire comment les systèmes politico-administratifs sont territorialement organisés. Il met en évidence leurs distinctes, mais complémentaires contributions : l'administration publique, en tant que sous-champ de la science politique, fait référence à l' « autorité », soit « les rôles formels conférés aux individus en leurs qualités officielles ${ }^{92}$, et à sa répartition formelle dans les différents niveaux de territoire gouvernementaux, alors que le politique, en tant que sous-champ de la science politique, prête « attention à l'autorité et au pouvoir, ainsi qu'aux interactions complexes entre les deux », mais son « inattention générale pour les dimensions territoriales du pouvoir et de l'autorité est peu louable ${ }^{93}$. De haut en bas, l'expression du pouvoir est le contrôle (ou la supervision). Puisque le contrôle étatique est inséparable des décisions des gouvernements locaux ${ }^{94}$, plus son intensité est grande, plus le degré d'autonomie locale est faible. Dans une perspective large des relations intergouvernementales, le contrôle du gouvernement central

90. MUELLER S., op. cit., 2011, p. 216.

91. HUTCHCROFT P.D., op. cit., 2001.

92. Ibidem, p. 26.

93. Ibidem, p. 27.

94. MARCOU G., op. cit., 1999. 
est entendu par Goldsmith comme «la définition des règles du jeu intergouvernemental ${ }^{95}$ et correspond à l'opposé de l'autonomie locale.

D'une part, un contrôle formel peut être opéré au travers d'un examen de la légalité des décisions locales en référence au cadre légal. La Charte met l'accent sur cette disposition pour que l'autonomie soit préservée ${ }^{96}$. Elle précise les limites et cherche à objectiver les conditions du contrôle administratif auquel les gouvernements locaux sont susceptibles de faire l'objet : les formes et les cas sont «prévus par la Constitution ou par la loi » (art. 8, al. 1) ; il ne « doit normalement viser qu'à assurer le respect de la légalité et des principes constitutionnels», mais «peut, toutefois, comprendre un contrôle de l'opportunité » (art. 8, al. 2); il doit être exercé « dans le respect d'une proportionnalité entre l'ampleur de l'intervention de l'autorité de contrôle et l'importance des intérêts qu'elle entend préserver » (art. 8, al. 3).

D'autre part, le contrôle opéré par les niveaux gouvernementaux supérieurs peut être exercé par divers moyens informels ${ }^{97}$. Premièrement, par le contrôle de l'autonomie financière du gouvernement local, à savoir les objets d'études du champ de la décentralisation fiscale, tels que la fixation des taux d'imposition, la discrétion pour faire des dépenses, l'accès à l'emprunt et les modalités du système de transfert. S'agissant de ce dernier objet, de nombreux auteurs ont souligné la contradiction inhérente à la décentralisation se trouvant dans le fait que la mise en place d'un système de subventionnement soit irrémédiablement liée à une augmentation du contrôle ${ }^{98}$. Deuxièmement, ce contrôle peut se traduire à travers le processus de régulation administrative par les façons dont certaines fonctions particulières peuvent être fournies. La supervision administrative examine dans ce cas l'opportunité des décisions locales, alors qu'elle se cantonne à un contrôle de légalité dans le cas d'un gouvernement local plus autonome. Troisièmement, les niveaux gouvernementaux supérieurs peuvent exercer un contrôle sur l'accès des gouvernements locaux vers les échelons supérieurs, lorsque celui-ci est garanti par un préfet nommé et non pas élu localement, à savoir « un fonctionnaire de l'État central dont les fonctions comprennent la supervision des actions du gouvernement local ${ }^{99}$, ainsi

95. GOLDSMITH M., « Central Control over Local Government - A Western European Comparison », Local Government Studies, vol. 28, n 3, 2002, p. 91.

96. CONSEIL DE L'EUROPE, op. cit., 1994.

97. GOLDSMITH M., op. cit., 2002.

98. PRUD'HOMME R., « On the Dangers of Decentralization », Policy Research Working Paper 1252, The World Bank, Washington D. C., 1994 ; FLEURKE F., WILLEMSE R., op. cit., 2004 ; GURR T.R., KING D.S., op. cit., 1987 ; OATES W.E., op. cit., 1999.

99. PAGE E.C., op. cit., 1991, p. 28. 
que par la sélection, par les responsables du parti aux niveaux supérieurs, des candidats en lice pour les élections locales ${ }^{100}$.

Pourtant, les préfets et les politiciens - entre autres acteurs locaux peuvent également jouer un rôle dans la défense des intérêts locaux vers les niveaux gouvernementaux supérieurs, ce que Page a appelé le «localisme politique » : «contrairement au [localisme juridique], les élites locales peuvent aussi influencer les politiques publiques d'une manière très différente en recourant à leur autorité politique, en tant que représentants démocratiquement légitimes de la localité, ou d'une partie importante de sa population, pour influencer les décisions nationales dans le cas où elles affectent la localité $\gg{ }^{101}$. D'après l'auteur, des canaux indirects et directs d'accès d'un gouvernement local aux niveaux plus élevés de gouvernement existent et offrent des opportunités pour les autorités locales de façonner les services publics.

La représentation indirecte se déroule collectivement au travers du lobbying corporatiste. Ainsi, plusieurs dimensions peuvent expliquer les forces et faiblesses des groupes d'intérêts locaux dans les pays, comme l'existence d'une association faîtière pour les gouvernements locaux, son intégration, sa cohésion interne, son autorité, ou encore son impact observé sur la politique nationale ${ }^{102}$. La représentation directe, qui offre la plus grande portée, se réfère à diverses arènes formelles d'interaction, parmi lesquelles l'expérience des législateurs et le cumul des mandats sont les plus importants ${ }^{103}$. Le problème avec une telle analyse statistique des expériences politiques, toujours selon Page ${ }^{104}$, est que la situation antécédente peut être confondue avec la motivation actuelle. Les autres indicateurs de l'accès vertical peuvent être l'existence d'une seconde chambre pour représenter le territoire local ${ }^{105}$, l'obligation de consulter les autorités locales «au cours des processus de planification et de décision pour toutes les questions qui les concernent directement ${ }^{106}$, l'existence d'outils d'influence de démocratie directe à disposition des gouvernements locaux, tels que l'initiative ou le référendum, ou encore la possibilité de choisir les candidats et les caractéristiques des systèmes électoraux (système de représentation, nombre et taille des circonscriptions, etc.) pour l'élection des représentants

100. RODDEN J., « Comparative Federalism and Decentralization: On Meaning and Measurement », in Comparative Politics, vol. 36, n 4, 2004, p. 481-500.

101. PAGE E.C., op. cit., 1991, p. 5.

102. Ibidem.

103. Ibidem.

104. Ibidem.

105. LIJPHART A., Democracies: Patterns of Majoritarian and Consensus Government in Twenty-one Democracies, New Haven, Yale UP, 1984.

106. CONSEIL DE L'EUROPE, op. cit., 1985a, art. 4, al. 6. 
à l'échelon local, mais aussi aux niveaux supérieurs (préfectoral, régional, national) ${ }^{107}$.

En somme, le degré d'influence qu'un gouvernement local est en mesure d'exercer directement ou indirectement sur les niveaux gouvernementaux supérieurs est également un facteur important de l'autonomie locale, puisqu'il peut permettre de changer le processus de décision des politiques publiques et les conditions de leur fourniture.

\section{Les études empiriques évaluant l'autonomie locale qualitativement et quantitativement dans une perspective comparative}

L'autonomie locale, appréhendée comme un concept relatif de type vertical, a été empiriquement évaluée en comparant ses modalités dans une sélection de pays types ${ }^{108}$. Pour ce faire, Page et Goldsmith ${ }^{109}$ ont résumé les limites imposées à un gouvernement local par les niveaux gouvernementaux supérieurs sous trois rubriques. Premièrement, il est question de la gamme des fonctions pour lesquelles le gouvernement local est responsable, mesurée par les dépenses locales en pourcentage du total des dépenses gouvernementales et l'emploi local en pourcentage des effectifs gouvernementaux totaux. Deuxièmement, les auteurs relèvent la portée de la discrétion, dans l'exercice de ces fonctions, définie comme «la capacité des acteurs au sein d'un gouvernement local de prendre des décisions sur le type et le niveau des services qu'il fournit, en tenant compte des obligations statutaires et du cadre administratif formel pour la prestation de services locaux, et de la façon dont ce service est fourni et financé $\gg^{110}$. Celle-ci peut être déterminée, selon les auteurs, par le cadre légal, la surveillance administrative et les compétences financières - à savoir la mesure dans laquelle les gouvernements locaux peuvent financer leurs opérations grâce à leurs propres revenus. La troisième catégorie de limite concerne le degré d'accessibilité ou d'influence du gouvernement local sur les niveaux

107. MUELLER S., op. cit., 2011 ; HUTCHCROFT P.D., op. cit., 2001 ; RODDEN J., op. cit., 2004 ; HORBER-PAPAZIAN K., L'intervention des communes dans les politiques publiques, PhD Thesis, Lausanne, Switzerland, École Polytechnique Fédérale de Lausanne, 2004 ; KUHLMANN S., WOLLMANN H., op. cit., 2014 ; DESCHOUWER K., «Political Parties as Multi-Level Organizations », in KATZ R.S., CROTTY W.J., Handbook of Party Politics, London, Sage Publications, 2006, p. 291-300 ; RIKER W., op. cit., 1975.

108. Pour une revue de littérature complète des études comparant les systèmes de gouvernement local entre les pays, voir WOLMAN, op. cit., 2008 ou KUHLMANN, WOLLMANN, op. cit., 2014.

109. PAGE E.C., GOLDSMITH M., op. cit., 1987. Voir aussi GOLDSMITH M., op. cit., 1995 ; GOLDSMITH M., PAGE E.C., Changing Government Relations in Europe: from Localism to Intergovernmentalism. London, Routledge, 2010.

110. PAGE E.C., GOLDSMITH M., op. cit., 1987, p. 5. 
gouvernementaux supérieurs. Comparant sept pays unitaires, Page ${ }^{111}$ est allé plus loin quantitativement en plusieurs points : en ce qui concerne le barème juridique, il a mesuré le poids respectif des subventions totales et spécifiques ; s'agissant du barème politique, il a mesuré l'adhésion visée et effective des groupes nationaux d'intérêts locaux et le pourcentage des politiciens nationaux qui ont précédemment été élus à l'échelon local. À ce titre, il convient de noter qu'un degré élevé de localisme politique n'implique pas un degré élevé de localisme juridique ${ }^{112}$.

Après avoir expliqué les raisons pour lesquelles elle ne prenait pas en compte la dimension horizontale de l'autonomie locale, Vetter ${ }^{113}$ est partie du principe que la dimension politique de l'autonomie locale développée par Page et Goldsmith ${ }^{114}$ et Page ${ }^{115}$ servait principalement à des fins clientélistes pour satisfaire les intérêts spécifiques locaux et, par conséquent, ne devait pas être prise en considération pour mesurer l'autonomie locale. Elle s'est donc concentrée plutôt sur les deux aspects du localisme juridique, à savoir l'éventail des fonctions exercées localement et la portée de la discrétion - les fonctions étant mesurées par les dépenses locales, et la discrétion l'étant par la structure des recettes locales (impôts, taxes, frais et allocations non spécifiques), ainsi que par la mention explicite du gouvernement local et de la protection financière dans la Constitution ${ }^{116}$.

Ajoutant pour leur sélection de pays une dimension basée sur la structure étatique (unitaire ou fédérale) à la typologie de Hesse et Sharpe ${ }^{117}$, Denters et Rose ${ }^{118}$ sont allés au-delà de l'analyse de la prestation des services. Ils ont mis en évidence les tendances relatives à l'étendue des responsabilités, à l'adoption de mesures de management interne (NGP) et externe (relations collaboratives), au renforcement de l'exécutif politique local et à la gouvernance locale démocratique. Stoker ${ }^{119}$ a fait valoir que les études comparatives de la gouvernance locale étaient en grande partie « occidentalocentrées » et essentiellement concernées par les différences institutionnelles formelles. Il a alors développé une typologie des rôles sociétaux que les systèmes de gouvernement local peuvent adopter: identité locale,

111. PAGE E.C., op. cit., 1991.

112. Ibidem.

113. VETTER A., op. cit., 2007.

114. PAGE E.C., GOLDSMITH M., op. cit., 1987.

115. PAGE E.C., op. cit., 1991.

116. VETTER A., op. cit., 2007.

117. HESSE J.J., SHARPE L.J., « Local Government in International Perspective: Some Comparative Observations », in HESSE J. (ed.), Local government and Urban Affairs in International Perspective, Baden-Baden, Nomos, 1991, p. 603-621.

118. DENTERS B., ROSE L.E., Comparing Local Governance: Trends and Developments, Basingstoke, Palgrave Macmillan, 2005.

119. STOKER G., op. cit., 2010. 
développement économique, fourniture de protection sociale et coordination des modes de vie. Si les gouvernements locaux sont considérés comme des organismes publics ${ }^{120}$, la conceptualisation et l'opérationnalisation de l'autonomie organisationnelle de Verhoest et al. ${ }^{121}$ ne peuvent être négligées. Quatre formes différentes d'autonomie organisationnelle y sont discernées : les autonomies structurelle, financière, juridique et interventionnelle. Ces différentes dimensions sont liées au concept de contrôle, considéré comme l'inverse de l'autonomie, et sont opérationnalisées pour constituer des indices. Ceux-ci ont été utilisés pour comparer l'autonomie des agences entre pays, mais pourraient très facilement être appliqués pour comparer l'autonomie des gouvernements locaux entre ces mêmes pays ${ }^{122}$.

Dans la «nouvelle approche comparative des compétences des collectivités locales dans les États membres du Conseil de l'Europe» développée par Marcou ${ }^{123}$, l'autonomie locale est définie comme la liberté avec laquelle une compétence est exercée et dépend avant tout des pouvoirs destinés à son exercice ainsi que des moyens nécessaires à sa mise en œuvre. Sur cette base, l'auteur compare les pouvoirs, les ressources et les compétences et développe une méthode pour analyser systématiquement le degré d'autonomie locale. Les critères «type de compétence », « exercice d'un pouvoir réglementaire », " décisions administratives individuelles », « gestion » et « contrôle » sont appliqués aux autorités locales des pays de l'échantillon pour leurs compétences en matière d'urbanisme uniquement, mais il est spécifié qu'ils peuvent également être employés pour d'autres fonctions ${ }^{124}$. D'autres études juridiques comparatives se sont focalisées sur les statuts (constitutionnels) des gouvernements locaux, leurs compétences juridiques ou la nature des relations avec les gouvernements supérieurs dont ils dépendent ${ }^{125}$. En raison de la nature du droit, ces études sont presque nécessairement qualitatives. Sceptiques vis-à-vis des indicateurs légaux et financiers, Fleurke et Willemse ${ }^{126}$ ont développé une approche axée sur la prise de décision afin de montrer la réelle pratique de cette dernière par

120. RHODES R.A.W., Control and Power in Central-Local Government Relations, Aldershot, GB, Gower and Brookfield VT, Ashgate, 1983.

121. VERHOEST K., PETERS G.B., BOUCKAERT G., VERSCHUERE B., «The Study of Organizational Autonomy: A Conceptual Review », Public Administration and Development, vol 24, $\mathrm{n}^{\circ} 2$ 2, 2004, p. 101-118.

122. Ibidem.

123. MARCOU G., op. cit., 2010, p. 1.

124. Ibidem, 2010, p. 59 et ss.

125. MORENO A.-M., Local Government in the Member States of the European Union: A Comparative Legal Perspective, Madrid, Instituto Nacional de Administración Publica, 2012 ; LOUGHLIN M., « The Constitutional Status of Local Government », in PRATCHETT L., WILSON D., Local Democracy and Local Government, London, MacMillan, 2001, p. 38-62 ; NORTON A., « West European Government in Comparative Perspective », in BATLEY R., STOKER G., Local government in Europe. Trends and developments, London, MacMillan, 1991, p. 21-40.

126. FLEURKE F., WILLEMSE R., op. cit., 2004. 
le gouvernement local. L'autonomie d'un gouvernement local est alors mesurée avec trois dimensions: son degré de liberté de choix politique, de dépendance à l'égard d'autres autorités et d'initiative pour déterminer son propre ordre du jour. La méthode est uniquement appliquée aux municipalités néerlandaises, mais il est suggéré par les auteurs qu'elle peut être utilisée dans d'autres contextes.

Do Vale ${ }^{127}$ compare l'autonomie infranationale sur trois continents en estimant son évolution dans les pays les plus décentralisés, respectivement le Brésil, l'Inde et l'Afrique du Sud. Différentes périodes sont analysées et les trois variables définies mesurent les degrés de changement que les pays connaissent au fil du temps. L'autonomie fiscale est appréhendée par des indicateurs traditionnels de la décentralisation fiscale, l'autonomie administrative par la part des employés du secteur public, et l'autonomie institutionnelle par la fréquence des réunions des instances gouvernementales ainsi que par le nombre de vetos institutionnels existant à l'échelon infranational. Les mesures quantitatives sont ici transformées en indicateurs qualitatifs de la décentralisation. Utilisant le terme «décentralisation» au lieu d' «autonomie locale », Sellers et Lidström ${ }^{128}$ ont conceptualisé la relation entre les niveaux gouvernementaux local et supérieur, en englobant à la fois les capacités et la surveillance des actions locales, dont les modalités sont liées à divers types d'États providences. La comparaison repose ici sur des indicateurs quantitatifs et qualitatifs codés quantitativement. Une grande variété de sources est mobilisée, y compris le suivi par pays effectué par le Conseil de l'Europe ${ }^{129}$. Les capacités locales sont mesurées au travers de deux indicateurs fiscaux (part des dépenses et des recettes) et trois indicateurs politico-administratifs (protections constitutionnelles, représentation corporative et part de l'emploi public). Il est intéressant de noter qu'en raison de la complexité qu'engendrerait un examen sectoriel, la part de l'emploi public est utilisée "comme approximation pour la répartition relative des pouvoirs ainsi qu'indications directes des capacités locales relatives ${ }^{130}$. Bien que plus détaillés, les indicateurs de surveillance ressemblent à l'inverse des indicateurs des capacités locales.

127. DO VALE H.F. « Comparing and Measuring Subnational Autonomy across Three Continents », Lex Localis, vol. 13, n 3, 2015, p. 741-764.

128. SELLERS J., LIDSTRÖM A., «Decentralization, Local Government, and the Welfare State », Governance: An International Journal of Policy, Administration and Institutions, vol. 20, $\mathrm{n}^{\circ} 4,2007$, p. 609-632.

129. Le Congrès des pouvoirs locaux et régionaux du Conseil de l'Europe surveille régulièrement la situation de la démocratie locale et régionale dans les États membres du Conseil de l'Europe. Les pays sont évalués tous les cinq ans. Ainsi, plus d'une cinquantaine de rapports nationaux ont été rédigés depuis 1995. Ceux-ci peuvent donner un premier aperçu de la situation de ces pays, mais ne produisent pas véritablement de données comparables, car certains d'entre eux ne sont pas focalisés sur le niveau local spécifiquement ou peuvent comporter une certaine dimension politique.

130. SELLERS, LIDSTRÖM, op. cit., 2007, p. 618. 
Des indices ont été développés non seulement dans une perspective comparative internationale, mais aussi pour une comparaison entre les régions d'un pays fédéral. Comparant l'autonomie locale entre les États américains, Wolman et al. ${ }^{131}$ se sont basés sur l'indice de centralisation unidimensionnel développé par Stephens ${ }^{132}$ pour définir l'autonomie au travers de trois dimensions: l'importance du gouvernement local dans l'économie et le système intergouvernemental, la discrétion du gouvernement local pour déterminer ce qu'il va faire sans contrainte excessive de la part des niveaux gouvernementaux supérieurs, et la capacité du gouvernement local à le faire. Trois aspects sont marquants dans leur indice de 21 variables. Premièrement, les dépenses locales directes exprimées en fonction du produit intérieur brut (PIB) et l'emploi local (consistant à l'emploi public) sont les variables mesurant l'importance. Deuxièmement, la discrétion englobe la portée juridique d'un gouvernement local, la responsabilité structurelle et fonctionnelle, ainsi que les contraintes sur les recettes (fiscales). Troisièmement, le personnel du gouvernement local par rapport à la population locale et la diversité des sources de revenu local mesurent la capacité. Évaluant comparativement quant à lui le degré de décentralisation des cantons suisses, Mueller ${ }^{133}$ établit une distinction entre les dimensions de la politique liées au système politique et aux politiques publiques, et pousse encore plus loin l'analyse des relations de pouvoir. La première dimension (polity) a pour originalité l'inclusion d'une mesure de l'autonomie perçue du secrétaire local, associée à un indice juridique existant. La deuxième (policy) comprend des mesures fiscales, administratives et relatives au personnel. La troisième (politics) reflète la complexité des relations intergouvernementales entre les cantons suisses, répartie en sept indicateurs (voir la sous-section «l'approche politique des relations intergouvernementales »). Quelques remarques doivent être formulées au sujet de ces deux études. Tout d'abord, certaines variables sont spécifiquement liées au contexte national, à l'exemple de la règle de Dillon. Ensuite, autant des indicateurs qualitatifs que quantitatifs sont utilisés. Enfin, l'unité d'analyse est plutôt le gouvernement régional, dont on analyse le degré de décentralisation, que le gouvernement local spécifiquement.

Dans les études qui visent à mesurer quantitativement le degré de décentralisation d'un gouvernement, la distinction est souvent opérée entre les dimensions politique, administrative et fiscale de la

131. WOLMAN H., MCMANMON R., BELLAND M., BRUNORI D., «Comparing Local Government Autonomy Across States », in BELL M.E., BRUNORI D., YOUGMAN J., The Property Tax and Local Autonomy, The Lincoln Institute, 2010, p. 69-114.

132. STEPHENS G.R., "State Centralization and the Erosion of Local Autonomy », Journal of Politics, vol. 36, $\mathrm{n}^{\circ}$ 1, 1974, p. 44-76.

133. MUELLER S., op. cit., 2015. 
décentralisation ${ }^{134}$. Afin de mesurer précisément in fine le degré de proximité entre le gouvernement et le peuple, Ivanyna et Shah ${ }^{135}$ ont élaboré une opérationnalisation poussée, dont l'une des innovations principales consiste en la pondération des scores effectuée à chaque stade. Des choix sont donc délibérément faits afin d'octroyer davantage de poids à certains aspects en particulier, impliquant une pénalisation - ou au contraire une favorisation - de certains pays, que ce soit dans un premier temps pour la création des indices de décentralisation fiscale, politique et administrative, dans un deuxième temps pour la construction de l'indice global de décentralisation, ou enfin pour le développement de l'indice final de proximité gouvernementale ${ }^{136}$.

Enfin, les études sur le fédéralisme et la décentralisation analysent la répartition de l'autorité entre les niveaux régional et national. Examinant les variations entre les États en ce qui concerne leur échelon régional respectif, Hooghe et al. ${ }^{137}$ opèrent la distinction entre les éléments concernant le degré d'autorité (signifiant l'autonomie formelle) d'une région sur les personnes qui vivent sur son territoire - règles exclusives - et l'influence des régions dans le façonnement du processus décisionnel national - règles communes. Ces deux dimensions sont alors divisées en quatre sous-dimensions qui permettent de quantifier l'autorité régionale dans 81 pays entre 1950 et 2010. Il convient de noter que ces huit variables constitutives de l'Indice d'Autorité Régionale (RAI) n'ont pas toutes la même échelle. Dans un processus de validation du RAI, Schakel ${ }^{138}$ compare le RAI avec d'autres indices de décentralisation institutionnelle ${ }^{139}$ et montre qu'il y a convergence. Même si ces autres indices de décentralisation ne se concentrent pas spécifiquement

134. SCHNEIDER A., op. cit., 2003 ; FALLETI T.G., op. cit., 2005 ; IVANYNA M., SHAH A., « How Close is your Government to its People? Worldwide Indicators on Localization and Decentralisation », Policy Research Working Paper, 6139, The World Bank, 2012.

135. IVANYNA M., SHAH A., op. cit., 2012.

136. Les données utilisées dans le cadre de cette étude proviennent de diverses bases de données sur les gouvernements locaux et la décentralisation, dont nombre d'entre elles ont été élaborées ces dernières années. L'observatoire mondial de la démocratie locale et de la décentralisation (GOLD selon l'acronyme anglais) de Cités et Gouvernements Locaux Unis (CGLU) produit des rapports qui incluent des profils nationaux décrivant qualitativement les structures territoriales, politiques, financières et de management des gouvernements locaux. La base de données de la Banque Mondiale (BM) fournit des indicateurs fiscaux quantitatifs basés sur des statistiques des finances publiques du Fonds Monétaire International (FMI) et des indicateurs qualitatifs sur la décentralisation fiscale et administrative. Il existe également une quantité considérable de données produites par l'OCDE. Le principal problème de ces sources est qu'elles concernent surtout les aspects financiers de l'autonomie locale et qu'elles n'opèrent souvent aucune distinction entre les niveaux infranationaux.

137. HOOGHE L., MARKS G., SCHAKEL A., CHAPMAN H., $\quad$ OSTERKATZ S., NIEDZWIECKIAND S., SHAIR-ROSENFIELD S., Measuring Regional Authority: A Postfunctionalist Theory of Governance, vol. I, Oxford, Oxford University Press, 2016.

138. SCHAKEL A.H., "Validation of the Regional Authority Index », Regional and Federal Studies, vol. 18, n 2-3, 2008, p. 143-166.

139. BRANCATI D., «Decentralization: Fueling or Dampening the Flames of Ethnic Conflict and Secessionism », International Organization, vol. 60, $\mathrm{n}^{\circ} 3$, 2006, p. 651-685 ; HOOGHE L., 
sur le niveau local, leurs approches, opérationnalisations et résultats sont également intéressants dans la perspective de cet article.

En résumé, il n'y a pas une théorie de l'autonomie locale, mais différentes approches axées sur des aspects spécifiques des dimensions du concept, multiples, mais interconnectées. En outre, les études analysant l'autonomie locale dans une perspective comparative combinent certaines dimensions de manière très variable. Enfin, seules quelques-unes adoptent une perspective longitudinale, pondèrent les variables, ont clairement et spécifiquement pour unité d'analyse le gouvernement local, ou encore différencient leur jugement en fonction des politiques publiques.

\section{Conceptualisation, méthodologie et création de l'IAL}

\section{Définition et conceptualisation de l'autonomie locale}

En se basant sur les considérations théoriques précédentes et la Charte - dans la mesure où le but est d'appliquer notre indice au contexte européen -, l'autonomie locale est appréhendée en tant que phénomène multidimensionnel, considéré à la fois comme un droit et une capacité. En effet, comme l'approche légaliste l'a montré, l'autonomie locale implique un droit et des pouvoirs décisionnels pour la gestion des affaires publiques, inclus dans un cadre juridique définissant les statuts formels des gouvernements locaux et les modalités de protection juridique de ces derniers. En lien avec l'étendue des compétences fonctionnelles formelles se trouve la gamme des services pour lesquels les gouvernements locaux sont responsables et les dépenses y relatives (output). Toutefois, pour rendre l'autonomie effective, un gouvernement local doit également avoir la capacité de les fournir concrètement. Par conséquent, l' autonomie locale est liée, d'une part, aux ressources financières dont les gouvernements locaux disposent de manière indépendante (input), et, d'autre part, à leurs capacités administratives et aux possibilités qu'ils ont de créer, organiser et entretenir leurs arènes politiques indépendamment (auto-gouvernance). Enfin, les relations politiques, qui existent dans le jeu de pouvoir intergouvernemental, sont également liées à l' autonomie locale dans le sens du contrôle et de l'accès.

Par conséquent, en se basant sur la définition de Page ${ }^{140}$, l'autonomie locale est définie comme « Le [droit et la] capacité des gouvernements locaux

MARKS G., Multi-level Governance and European Integration, Lanham, Rowman and Littlefield, 2001 ; TREISMAN D., op. cit., 2002.

140. PAGE E., op. cit., 1982, p. 21. 
[dans un cadre juridique défini] de prendre des décisions sur les services qu'ils fournissent [et les ressources financières nécessaires] sans ingérence du centre ${ }^{141} »$. Dès lors, sept dimensions constitutives de l'autonomie locale sont identifiées :

- L'autonomie juridique : les statuts formels des gouvernements locaux et les modalités de protection juridique de ceux-ci ;

- La discrétion politique: la distribution générale du pouvoir et les compétences décisionnelles effectives attribuées aux gouvernements locaux pour la fourniture des services ;

- Le champ d'exécution: la gamme des services pour lesquels les gouvernements locaux sont responsables ;

- L'autonomie financière : les ressources financières disponibles pour les gouvernements locaux et la possibilité de décider librement de leurs sources ;

- L'autonomie organisationnelle: la libre organisation des arènes politiques et de l'administration propres aux gouvernements locaux ;

- La non-ingérence : l'étendue de la liberté laissée aux gouvernements locaux dans le cadre du contrôle effectué ;

- L'accès : le degré d'influence des gouvernements locaux sur les décisions politiques prises par les niveaux gouvernementaux supérieurs.

L'importance relative de ces sept dimensions est cependant difficile à établir, car elle dépend de la perspective adoptée et des raisons pour lesquelles le concept est utilisé dans le cadre de recherches empiriques.

\section{Méthodologie de l'analyse empirique et analyse factorielle}

En 2014, la Direction générale de la politique régionale et urbaine de la Commission européenne a lancé un appel d'offres pourévaluer et documenter les évolutions dans le degré de décentralisation de 39 pays européens de 1990 à 2014. Le document central de cette étude comparative d'ampleur, permettant de passer outre la complexité liée aux diverses unités existant au niveau local et aux asymétries institutionnelles présentes en Europe ${ }^{142}$,

141. «Le centre » est entendu dans cet article comme les niveaux gouvernementaux supérieurs, dans la mesure où les pays fédéraux sont également pris en compte, alors que Page (1991) était davantage intéressé aux pays unitaires.

142. Il est nécessaire de souligner que la question importante de la spatialité de la prise de décision - à savoir l'échelle à laquelle les décisions des autorités locales sont réellement prises - n'est pas abordée ici, conformément aux limitations fixées pour appréhender comparativement et empiriquement l'autonomie locale. Il en reste néanmoins qu'à ce stade, l'unité d'analyse doit être définie : l'autonomie locale se réfère au gouvernement local (communément appelé «municipalité »), unité administrative 
est un livre de codage standardisé intégrant onze variables. Celles-ci ont été choisies et opérationnalisées sur la base de considérations théoriques et empiriques, et en respectant autant que possible la méthodologie du RAI mentionné supra ${ }^{143}$. L'inclusion dans le livre de codage d'aspects ne pouvant pas être mesurés avec la méthode choisie - le « jugement d'experts ${ }^{144}-$ ne faisant aucun sens, une partie des indices mis en évidence dans le deuxième chapitre de ce présent article n'y sont pas pris en compte. Néanmoins, les onze variables suivantes permettent une appréciation globale de l'autonomie locale dans les pays analysés :

- La profondeur institutionnelle: la mesure dans laquelle les gouvernements locaux sont formellement autonomes et peuvent choisir les tâches qu'ils désirent effectuer ;

- Le champ de responsabilités : l'étendue des services (tâches) pour la fourniture desquels les gouvernements locaux sont responsables (que ce soit au moyen des ressources financières ou de la main-d'œuvre propres) ;

- La discrétion politique effective: la mesure dans laquelle les gouvernements locaux ont une réelle influence sur ces services (peuvent décider de leurs aspects) ;

- L'autonomie fiscale : la mesure dans laquelle les gouvernements locaux peuvent indépendamment taxer leur population ;

- Le système de transferts financiers : la proportion des transferts financiers inconditionnels par rapport aux transferts financiers totaux reçus par les gouvernements locaux ;

- L'autosuffisance financière : la proportion des recettes des gouvernements locaux provenant de sources locales (impôts, taxes, frais) ;

- L'autonomie en matière d'emprunt: la mesure dans laquelle les gouvernements locaux sont en mesure d'emprunter ;

située au plus bas de l'architecture étatique et classée en-dessous d'une province, d'une région ou d'un État (généralement le niveau « LAU 2 » selon la nomenclature des unités territoriales statistiques de la Commission européenne). Lorsque les gouvernements locaux jouissent d'un degré d'autonomie différent au sein d'un même pays, ces asymétries ont été prises en compte en opérant une pondération par la population des scores attribués selon les règles suivantes : «dans un pays unitaire où toutes les municipalités ont le même degré d'autonomie, l'unité d'agrégation est le pays ; dans un pays unitaire disposant d'arrangements asymétriques, il existe différentes unités d'agrégation (par exemple les municipalités en général et les villes ayant des compétences spéciales); dans les pays fédéraux où toutes les municipalités ont le même degré d'autonomie, l'unité d'agrégation est le pays ; dans les pays fédéraux où le degré d'autonomie varie d'une entité fédérée à l'autre, les unités d'agrégation sont les entités fédérées » (LADNER A., KEUFFER N., BALDERSHEIM H., « Measuring Local Autonomy in 39 Countries (1990-2014) », Regional \& Federal Studies, vol. 26, n 3, 2016, p. 351 et ss).

143. LADNER A., KEUFFER N., BALDERSHEIM H., op. cit., 2016.

144. Ibidem, p. 328. 
- L'autonomie organisationnelle: la mesure dans laquelle les gouvernements locaux sont libres de décider de leurs propres organisation et système électoral ;

- La protection juridique: l'existence de moyens constitutionnels ou légaux pour faire valoir l'autonomie locale ;

- La supervision administrative : la mesure dans laquelle la supervision administrative des gouvernements locaux n'est pas contraignante ;

- L'accès $\mathrm{au}(\mathrm{x})$ gouvernement(s) supérieur(s) : la mesure dans laquelle les gouvernements locaux sont consultés pour influencer les décisions politiques prises par le gouvernement régional et/ou central.

Les opérationnalisations et échelles respectives de ces onze variables ne sont pas les mêmes (voir les troisième et cinquième colonnes du Tableau $\mathrm{A}$, en annexe). Celles-ci suivent en partie le livre de codage développé dans le cadre du RAI, toutefois, certaines modifications ont été apportées et des variables ajoutées, pour que l'analyse retranscrive au mieux les réalités locales des pays étudiés (voir la sixième colonne du Tableau $\mathrm{A}$, en annexe). À ce titre, il convient de préciser que les politiques publiques sélectionnées pour les variables «le champ de responsabilités » et «la discrétion politique effective » correspondent à celles dont les gouvernements locaux en Europe sont principalement responsables ${ }^{145}$.

Ayant rassemblé une large quantité d'information grâce à l'attribution d'un score pour les onze variables (pour les 39 pays étudiés et pour chaque année de la période couverte), une série d'analyses factorielles, avec un nombre imposé de facteurs, montrent empiriquement quelles variables sont liées les unes aux autres (voir Tableau 1). Cette technique de réduction combine les variables connexes dans un plus petit nombre de variables, pour qu'au sein de chaque facteur, chacune d'entre elles soit indépendante des autres ${ }^{146}$. Toutefois, le processus complet de réduction se base également sur les considérations théoriques et les écueils identifiés dans la littérature.

Les résultats des analyses factorielles montrent que trois variables restent seules lorsque le nombre de facteurs augmente: la protection juridique, l'accès $\mathrm{au}(\mathrm{x})$ gouvernement(s) supérieur(s) et l'autonomie organisationnelle. Ces variables mesurent donc des dimensions distinctes de l'autonomie locale. Ensuite, l'autonomie fiscale et l'autosuffisance financière sont ensemble sur le même facteur dans les quatre solutions testées, ce qui s'avère tout à fait logique puisque l'autonomie fiscale donne

145. MARCOU G., op. cit., 2010 ; LOUGHLIN J., HENDRIKS F., LIDSTRÖM A. (eds.), The Oxford Handbook of Local and Regional Democracy in Europe, Oxford: Oxford University Press, 2011 ; MORENO A.-M., op. cit., 2012.

146. WOLMAN H. et al., op. cit., 2010. 
aux gouvernements locaux un meilleur accès aux ressources financières propres. En ce qui concerne la question financière, il est intéressant de noter que le système de transferts financiers n'est pas lié à ces variables-ci, mais à la supervision administrative. Ce résultat empirique semble plutôt donner raison aux chercheurs ayant affirmé que les subventions affectées font l'objet d'un strict contrôle. Les variables « système de transfert financier » et «supervision administrative » seront donc analysées ensemble. Comme il l'a été dit dans le chapitre théorique, l'autonomie en matière d'emprunt est liée aux restrictions que les niveaux gouvernementaux supérieurs peuvent imposer dans la loi. Bien que l'autonomie en matière d'emprunt soit principalement corrélée avec la profondeur institutionnelle dans les solutions des analyses factorielles, elle peut contribuer à l'autonomie financière, comme le suggère l'art. 9 de la Charte. Cela peut se voir empiriquement au travers des coefficients (entre 0,24 et 0,32 ) sur les facteurs combinant l'autonomie fiscale et l'autosuffisance financière lorsque leur nombre total est de 5 à 7.

Le champ de responsabilités et la discrétion politique effective se trouvent sur le même facteur dans toutes les solutions testées. Cela signifie potentiellement que le principe d'équivalence fiscale ${ }^{147}$ est respecté : si les gouvernements locaux sont impliqués dans la fourniture de services au travers de leurs propres ressources financières et/ou de leur propre main-d'œuvre, ils disposent également de la possibilité de décider de certains de leurs aspects. Suivant les préoccupations souvent exprimées par les gouvernements locaux selon lesquelles ils n'ont seulement qu'à payer et exécuter (décentralisation administrative) sans pouvoir décider (décentralisation politique), ou les arguments scientifiques prônant que la fourniture de services et la décision politique effective sont des aspects distincts, ceux-ci seront plutôt analysés séparément. Par conséquent, la discrétion politique sera appréhendée en considérant la discrétion politique effective ainsi que la profondeur institutionnelle. Ce regroupement se légitime autant empiriquement que théoriquement: empiriquement, le coefficient de la profondeur institutionnelle sur le facteur combinant le champ de responsabilités et la discrétion politique effective est relativement élevé pour les solutions entre 5 et 7 facteurs (entre 0,38 et 0,45 ) ; théoriquement, les deux éléments sont relatifs à la discrétion politique, la profondeur institutionnelle concernant la distribution générale formelle du pouvoir et la discrétion politique effective touchant les compétences décisionnelles effectives relatives à des fonctions spécifiques.

147. OLSON M., « The Principle of 'Fiscal Equivalence': The Division of Responsibilities among Different Levels of Government », The American Economic Review, vol. 59, 1969, n² 2, p. 479-487. 
Tableau 1 : Analyses factorielles, avec un nombre imposé de facteurs $(5,6,7$ et 8$)$

\begin{tabular}{|c|c|c|c|c|c|c|c|c|c|c|c|c|c|c|}
\hline & 1 & 2 & 3 & 4 & 5 & & 1 & 2 & 3 & 4 & 5 & 6 & 7 & 8 \\
\hline $\begin{array}{l}\text { La profondeur } \\
\text { institutionnelle }\end{array}$ & .41 & .74 & .01 & .03 & .13 & & .29 & .06 & .03 & .24 & .07 & .29 & .21 & .81 \\
\hline $\begin{array}{l}\text { Le champ de } \\
\text { responsabilités }\end{array}$ & .86 & .29 & .21 & .02 & .08 & & .83 & .23 & .06 & .19 & -.01 & .24 & .04 & .20 \\
\hline $\begin{array}{l}\text { La discrétion } \\
\text { politique effective }\end{array}$ & .90 & .13 & -.03 & .06 & .05 & & .95 & -.04 & .06 & .09 & .05 & .03 & .09 & .10 \\
\hline L'autonomie fiscale & .30 & .09 & .87 & .11 & .01 & & .24 & .89 & -.02 & .16 & .09 & .18 & .00 & -.02 \\
\hline $\begin{array}{l}\text { Le système de } \\
\text { transferts financiers }\end{array}$ & .15 & .15 & .35 & -.36 & .69 & & .18 & .41 & .60 & -.30 & -.19 & -.10 & .01 & .44 \\
\hline $\begin{array}{l}\text { L'autosuffisance } \\
\text { financière }\end{array}$ & -.14 & .29 & .82 & -.18 & .18 & & -.12 & .81 & .13 & -.06 & -.19 & .06 & .33 & .15 \\
\hline $\begin{array}{l}\text { L'autonomie en } \\
\text { matière d'emprunt }\end{array}$ & -.01 & .71 & .32 & .30 & .04 & & .12 & .20 & .08 & .13 & .12 & .15 & .91 & .15 \\
\hline $\begin{array}{l}\text { L'autonomie } \\
\text { organisationnelle }\end{array}$ & .44 & .22 & .09 & .54 & .19 & & .24 & .08 & .17 & .89 & .10 & .04 & .12 & .15 \\
\hline $\begin{array}{l}\text { La protection } \\
\text { juridique }\end{array}$ & -.01 & .07 & -.06 & .84 & .02 & & .03 & -.05 & .08 & .09 & .98 & .02 & .10 & .03 \\
\hline $\begin{array}{l}\text { La supervision } \\
\text { administrative }\end{array}$ & .06 & .14 & -.03 & .37 & .83 & & .04 & -.02 & .88 & .27 & .16 & .18 & .09 & -.03 \\
\hline \multirow{2}{*}{$\begin{array}{l}\text { L'accès au }(\mathrm{x}) \\
\text { gouvernement(s) } \\
\text { supérieur(s) }\end{array}$} & .22 & .73 & .15 & .00 & .13 & & .19 & .19 & .14 & .04 & .02 & .90 & .15 & .19 \\
\hline & 1 & 2 & 3 & 4 & 5 & 6 & & 1 & 2 & 3 & 4 & 5 & 6 & 7 \\
\hline $\begin{array}{l}\text { La profondeur } \\
\text { institutionnelle }\end{array}$ & .38 & .73 & .01 & .13 & -.04 & .15 & & .45 & -.03 & .58 & .14 & .14 & .41 & -.03 \\
\hline $\begin{array}{l}\text { Le champ de } \\
\text { responsabilités }\end{array}$ & .85 & .29 & .21 & .08 & -.01 & .16 & & .85 & .24 & .10 & .08 & .18 & .25 & -.01 \\
\hline $\begin{array}{l}\text { La discrétion } \\
\text { politique effective }\end{array}$ & .90 & .14 & -.03 & .06 & .06 & .12 & & .93 & -.02 & .08 & .07 & .11 & .02 & .07 \\
\hline L'autonomie fiscale & .29 & .09 & .87 & .01 & .09 & .10 & & .25 & .90 & .01 & .03 & .11 & .16 & .07 \\
\hline $\begin{array}{l}\text { Le système de } \\
\text { transferts financiers }\end{array}$ & .19 & .15 & .35 & .73 & -.22 & -.24 & & .21 & .28 & .15 & .82 & -.16 & .02 & -.17 \\
\hline $\begin{array}{l}\text { L'autosuffisance } \\
\text { financière }\end{array}$ & -.15 & .28 & .82 & .19 & -.19 & -.03 & & -.13 & .77 & .36 & .27 & -.05 & .07 & -.20 \\
\hline $\begin{array}{l}\text { L'autonomie en } \\
\text { matière d'emprunt }\end{array}$ & -.05 & .71 & .32 & .02 & .18 & .26 & & .07 & .24 & .85 & .06 & .16 & .11 & .17 \\
\hline $\begin{array}{l}\text { L'autonomie } \\
\text { organisationnelle }\end{array}$ & .27 & .19 & .07 & .09 & .09 & .88 & & .29 & .09 & .22 & -.04 & .87 & .06 & .07 \\
\hline $\begin{array}{l}\text { La protection } \\
\text { juridique }\end{array}$ & .04 & .10 & -.05 & .03 & .97 & .09 & & .05 & -.04 & .12 & -.03 & .09 & .02 & .97 \\
\hline $\begin{array}{l}\text { La supervision } \\
\text { administrative }\end{array}$ & .00 & .13 & -.05 & .80 & .23 & .38 & & -.03 & -.06 & -.01 & .69 & .54 & .22 & .26 \\
\hline $\begin{array}{l}\text { L'accès au(x) } \\
\text { gouvernement(s) } \\
\text { supérieur(s) }\end{array}$ & .23 & .73 & .15 & .15 & .04 & -.04 & & .18 & .20 & .18 & .09 & .07 & .91 & .03 \\
\hline
\end{tabular}

Méthode d'extraction: Analyse en composantes principales; Méthode de rotation: Varimax avec normalisation Kaiser. $\mathrm{N}=966$ (39 pays, 25 années). 


\section{Création de l'IAL}

La création d'un indice d'autonomie locale (IAL) prend en compte le fait que tous les aspects de l'autonomie locale ne sont pas d'égale importance. La construction est ainsi réalisée en deux étapes : d'abord, la pondération des variables incluses dans les sept dimensions ; ensuite, la pondération des dimensions qui composent ensemble l'IAL. Cette approche a également été suivie par Ivanyna et $S_{h a h}{ }^{148}$; cependant, leur étude empirique est très différente de celle-ci en de nombreux points, tels que les objectifs, le contexte ou encore la méthode. Par conséquent, étant donné que la littérature existant sur le sujet n'est pas univoque sur le degré d'importance à conférer aux multiples dimensions constitutives de l'autonomie locale, des choix sont opérés, sur la base de considérations théoriques et empiriques ainsi que du jugement générique des experts impliqués dans cette recherche ${ }^{149}$. En ce qui concerne la pondération des onze variables, les experts ont qualifié de «très importantes » la discrétion politique effective, l'autonomie fiscale, l'autosuffisance financière et l'autonomie organisationnelle. Le champ de responsabilités et la protection juridique sont quant à eux considérés comme «importants», alors que la profondeur institutionnelle, l'autonomie en matière d'emprunt, le système de transferts financiers, la supervision administrative et l'accès au(x) gouvernement(s) supérieur(s) sont définis comme «peu importants » (voir la quatrième colonne du Tableau A, en annexe) ${ }^{150}$. Ainsi, les sept dimensions (D) sont construites (pour chaque année) comme suit :

- D_autonomie juridique $=100 / 3 *$ protection juridique ;

- D_discrétion politique $=100 / 15^{*}($ profondeur institutionnelle + $3 *$ discrétion politique effective) ;

- D_champ d'exécution $=100 / 4 *$ champ de responsabilités ;

- D_autonomie financière $=100 / 24 *(3 *$ autonomie fiscale + $3^{*}$ autosuffisance financière +1 *autonomie en matière d'emprunt) ;

- D_autonomie organisationnelle $=100 / 4 *$ autonomie organisationnelle ;

- D_non-ingérence $=100 / 6 *$ (système de transferts financiers + supervision administrative) ;

- D_accès $=100 / 3 *$ accès au $(\mathrm{x})$ gouvernement(s) supérieur(s).

148. IVANYNA M., SHAH A., op. cit., 2012.

149. LADNER A., KEUFFER N., BALDERSHEIM H., Local Autonomy Index for European countries (1990-2014). Release 1.0, Brussels, European Commission, 2015, p. 65.

150. En plus de la pondération relative des variables (une variable est pondérée, lorsque c'est le cas, en fonction de l'autre variable constitutive de la dimension donnée), les valeurs sont transformées à partir de leur échelle d'origine (voir la troisième colonne du Tableau $\mathrm{A}$, en annexe) en échelle graduée de 0 à 100 (100 étant le degré d'autonomie locale le plus élevé). 
Parmi les sept dimensions, les experts ont jugé que deux d'entre elles sont «très importantes » (discrétion politique et autonomie financière), deux d'entre elles sont « importantes » (champ d'exécution et autonomie organisationnelle) et trois d'entre elles sont «peu importantes » (autonomie juridique, non-ingérence et accès) (voir la deuxième colonne du Tableau $\mathrm{A}$, en annexe). Par conséquent, l'IAL est construit (pour chaque année comme suit :

$$
\begin{aligned}
\text { IAL }= & (1 * \text { D_autonomie juridique }+3 * \text { D_discrétion politique }+ \\
& 2 * \text { D_champ d'exécution }+3 * \text { D_autonomie financière }+ \\
& 2 * \text { D_autonomie organisationnelle }+1 * \text { D_non-ingérence }+ \\
& 1 * \text { D_accès }) / 13 .
\end{aligned}
$$

L'IAL construit, le chapitre suivant présente les principaux résultats obtenus pour les 39 pays européens couverts et leur évolution de 1990 à 2014, au niveau des sept dimensions constitutives de l'autonomie locale et de l'IAL.

\section{Principaux résultats}

\section{Au niveau des sept dimensions constitutives de l'autonomie locale}

En tant que concept multidimensionnel, l'autonomie locale est intéressante à analyser au travers de ses dimensions constitutives. Selon la construction des sept dimensions présentée supra, les 39 pays couverts ont en effet des scores très variés pour 2014 (voir Tableau B, en annexe), et il ressort que les pays européens présentent des profils nationaux d'autonomie locale très différents (voir Figure 1).

En ce qui concerne l'autonomie juridique, la Finlande (en tant qu'exemple des pays nordiques), l'Espagne (en tant qu'exemple des pays méditerranéens) et la Géorgie (en tant qu'exemple des pays d'Europe centrale et orientale) ont des scores qui correspondent à la moyenne, alors que la Suisse (en tant qu'exemple des pays centraux germanophones) a l'un des plus élevés.

S'agissant de la discrétion politique et du champ d'exécution, la Finlande est parmi les pays européens leaders, loin devant les trois autres pays, dont les scores se trouvent tous en dessous de la moyenne, à l'exception de celui de la Suisse pour le champ d'exécution. À ce propos, les gouvernements locaux suisses fournissent vraisemblablement une grande part des services, mais sans jouir nécessairement de la discrétion politique effective y relative. En comparaison, les gouvernements locaux nordiques sont forts, 
fournissent une large gamme de politiques publiques en pouvant décider de leurs modalités et semblent ne pas avoir besoin de moyens assurant la protection juridique de leur autonomie (la Norvège a le score minimum pour l'autonomie juridique).

En termes d'autonomie financière, la Suisse a le score maximum, devant les pays nordiques, les autres pays centraux germanophones ou les pays méditerranéens, ces derniers se trouvant juste au-dessus de la moyenne. Il ressort clairement que la Géorgie et les autres pays d'Europe centrale et orientale ne bénéficient pas du même degré d'autonomie financière et de ressources financières (l'Ukraine a le score minimum). Les résultats sont à peu près équivalents à l'égard de l'autonomie organisationnelle - la Suisse atteint le score de 100, la Finlande 75, l'Espagne 50 et la Géorgie 25 -, mais des extrapolations sont difficiles à formuler.

Figure 1 : Profils suisse, finlandais, espagnol et géorgien d'autonomie locale (7 dimensions, 2014)

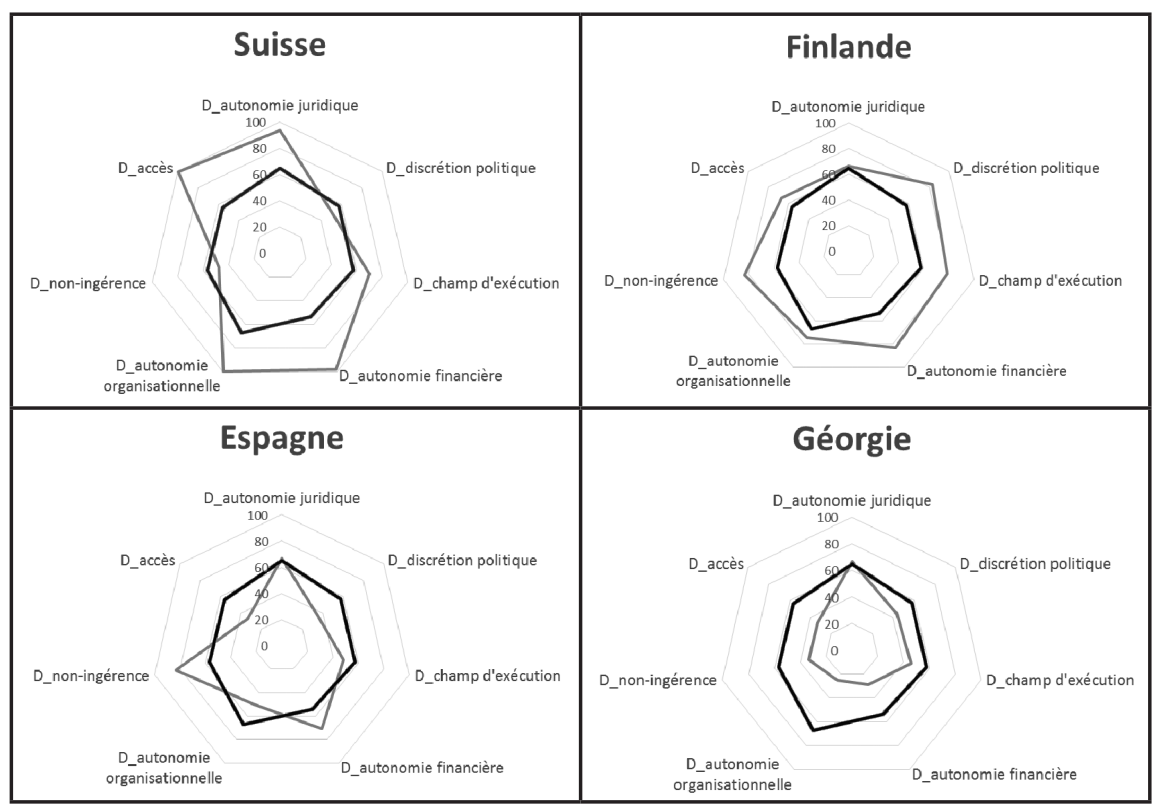

En gras : la moyenne des 39 pays couverts (2014).

Concernant la non-ingérence, les décisions locales et les transferts financiers de la Géorgie et des gouvernements locaux de nombreux autres pays du centre et de l'est de l'Europe semblent être soumis à une supervision forte. Les scores de la Finlande (ainsi que d'autres pays nordiques) et de l'Espagne sont les plus élevés, tandis que celui de la Suisse est inférieur à la moyenne, en partie en raison de la structure fédérale qui y prévaut. Cela se retrouve également dans le score élevé de la Suisse quant aux possibilités 
d'accès des gouvernements locaux, parmi les plus importantes en Europe, alors que pour cette dimension, le score de la Finlande se trouve près de la moyenne, et que ceux de la Géorgie et de l'Espagne représentent le minimum.

\section{Au niveau de l'IAL}

Les principaux résultats au niveau de l'IAL, calculé pour 2014, révèlent que les premiers rangs sont occupés par les pays nordiques, la Suisse, l'Allemagne et la Pologne, alors que les pays où les gouvernements locaux ont le degré d'autonomie le plus bas se situent au sud-est de l'Europe, à l'exception de l'Irlande (voir Figure 2). Plus globalement, cinq groupes peuvent être identifiés ${ }^{151}$ :

1. Un groupe composé des pays nordiques (la Finlande, l'Islande, le Danemark, la Suède et la Norvège) et de certains pays centraux (la Suisse, l'Allemagne et la Pologne), tous ayant un score d'autonomie locale supérieur à 69,55 ;

2. Les pays dont le score de l'IAL en 2014 se situe entre 60,78 et 69,55, à savoir le Liechtenstein, l'Italie, la Serbie, la France, la Bulgarie, la Lituanie, l'Autriche, la République tchèque et l'Estonie ;

3. Les pays où le degré d'autonomie locale est moyen (score de l'IAL entre 50,07 et 60,77), c'est-à-dire le Portugal, la Slovaquie, la Belgique, les Pays-Bas, la Macédoine, la Roumanie, la Croatie, le Luxembourg, la Lettonie et l'Espagne ;

4. Un groupe de pays dont le score de l'IAL en 2014 se situe entre 41,77 et 50,06, c'est-à-dire la Hongrie, l'Albanie, la Slovénie, l'Ukraine, la Grèce et le Royaume-Uni ;

5. Un groupe de pays où les gouvernements locaux jouissent d'un degré d'autonomie locale bas (le score pour 2014 est inférieur à 41,76), soit les pays du sud de l'Europe et ceux qui entourent la Mer noire (Chypre, la Turquie, la Géorgie, Malte et la Moldavie) ainsi que l'Irlande.

S'agissant des évolutions générales, les résultats montrent qu'une augmentation de l'autonomie locale a eu lieu entre 1990 et 2014, en particulier dans les nouvelles démocraties d'Europe centrale et orientale (voir Tableau $\mathrm{C}$, en annexe). Cela a eu lieu plus précisément jusqu'en 2009 (moy. $=58,62$ ), puisque le tableau d'ensemble montre une légère tendance à la centralisation depuis lors. La crise financière de 2007/08 n'est certainement pas étrangère à cette stabilisation de l'autonomie locale.

151. Cette classification repose sur l'algorithme Seuils naturels (Jenks). Les classes de seuils naturels dépendent des regroupements naturels inhérents aux données. Les bornes des classes désignées grâce à cette méthode permettent ainsi de regrouper au mieux des valeurs similaires et optimiser les différences entre les classes. 


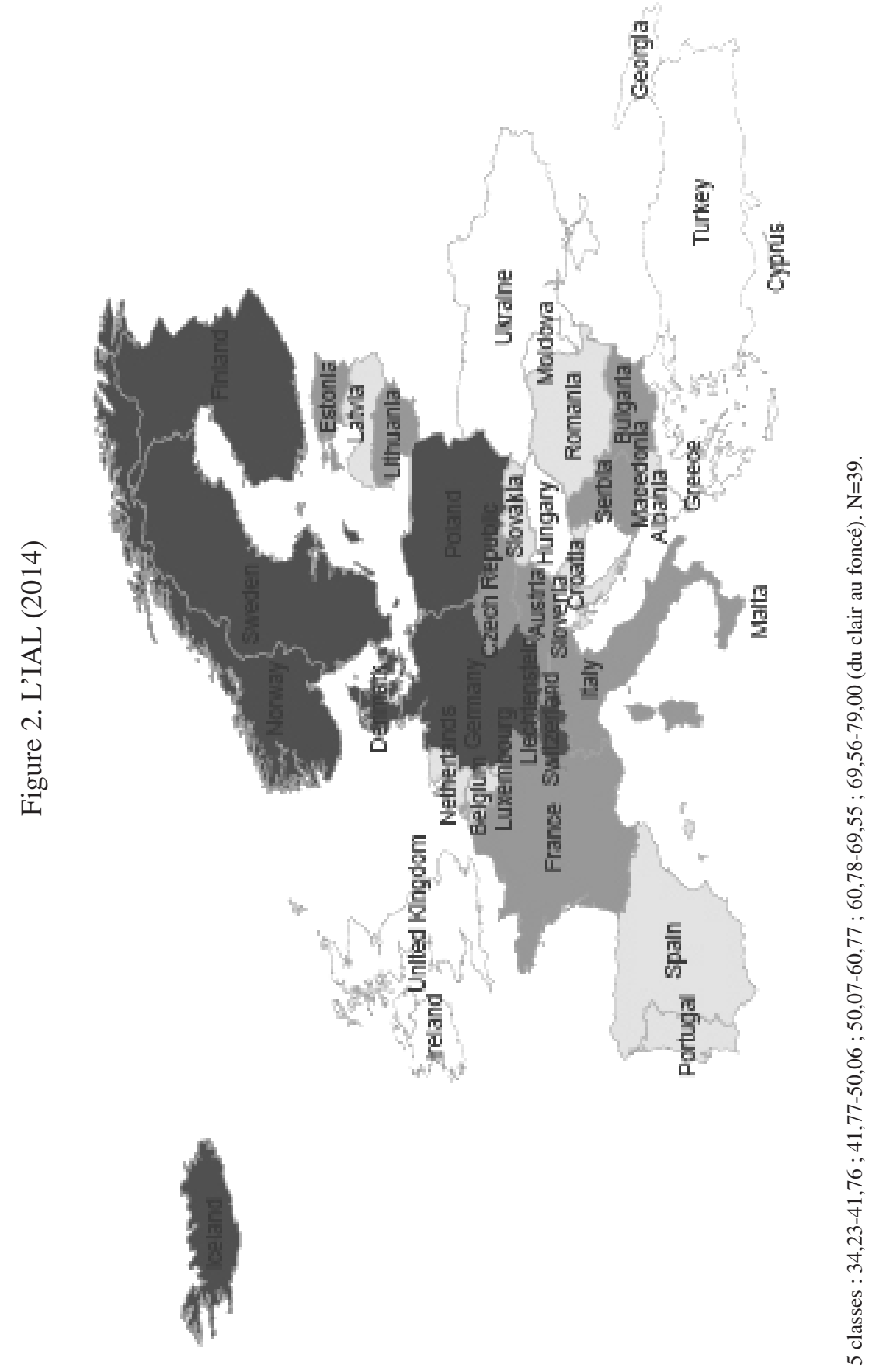




\section{Discussion et conclusion}

En s'appuyant sur les théories de la décentralisation et du gouvernement local, cet article propose un indice d'autonomie local légitimé théoriquement et applicable dans une perspective comparative. La revue de la littérature réalisée montre que l'autonomie locale est un concept complexe, multidimensionnel et traité par différentes approches. Celles-ci définissent différemment l'autonomie locale et chacune se concentre sur des aspects en particulier. Cependant, tout comme les dimensions de l'autonomie locale, ces approches sont interconnectées et il arrive parfois que leurs objets d'étude respectifs se chevauchent. Quant aux études empiriques évaluant l'autonomie locale dans une perspective comparative, elles combinent généralement les dimensions très différemment, sans leur conférer un degré d'importance respectif.

Après avoir défini l'autonomie locale et appliqué un livre de codage de onze variables à 39 pays européens pour la période allant de 1990 à 2014, les résultats empiriques, combinés avec les considérations théoriques, nous ont amenés à proposer le regroupement des variables en sept dimensions constitutives de l'autonomie locale ainsi qu'une procédure de pondération pour la construction d'un indice général de l'autonomie locale. L'IAL a été utilisé pour mesurer comparativement l'autonomie locale d'une manière globale à travers un grand nombre de pays et sur une longue période de temps, nous permettant ainsi de souligner les évolutions majeures en termes de décentralisation. Les principaux résultats montrent que les pays nordiques, la Suisse, l'Allemagne et la Pologne se classent tout en haut de l'IAL, mais que leurs scores respectifs sont différemment élevés en fonction des dimensions. Par ailleurs, une augmentation générale de l'autonomie locale a eu lieu entre 1990 et 2009, en particulier dans les nouvelles démocraties d'Europe centrale et orientale.

Pour parvenir à ces résultats, des choix ont bien entendu été opérés. Tout d'abord, l'autonomie locale a été considérée dans sa relation verticale à l'État. Dans d'autres recherches, peut-être des études nationales détaillées, la direction horizontale de la décentralisation et de l'autonomie locale ainsi que la conception de l'autonomie locale en tant que construction relationnelle complexe pourraient également être prises en compte. L'accent pourrait aussi être porté sur les explications des variations de l'autonomie locale dans le temps et en fonction des domaines politiques, ou sur la spatialité de la prise de décision. En ce qui concerne l'évaluation des impacts et conséquences de l'autonomie locale, nous espérons que l'IAL constituera un tremplin pour de nouvelles études comparatives portant sur les implications de l'autonomie locale pour la démocratie locale et/ou l'efficacité du gouvernement local. 
D'autre part, la définition de l'autonomie locale et son découpage en sept dimensions découlent de différents critères d'évaluation de l'autonomie locale existant dans la littérature consacrée. Pourtant, certains débats existent au sein même de ces approches et il peut y avoir des chevauchements entre ces dernières. La répartition des déterminants de l'autonomie locale proposée, en quatre approches, est donc une tentative de classification parmi d'autres possibles. En effet, d'autres aspects auraient pu être regroupés, à l'instar des capacités financières et administratives, de l'organisation politique et de l'accès, voire des compétences formelles et des responsabilités en matière de services. À cet égard, il pourrait être intéressant de pousser plus loin l'analyse, en se concentrant également sur les théories respectives des différentes disciplines.

Ensuite, toute construction d'indice exige la plus grande prudence dans le traitement des résultats qui s'ensuivent et suggère de regarder la situation dans son ensemble, dans la mesure où un simple changement d'indicateur peut conduire à un jugement complètement différent ${ }^{152}$. En outre, les critères d'évaluation de l'autonomie locale sont liés aux fondements normatifs d'un gouvernement local. Par conséquent, l'inclusion de certaines variables et leur pondération impliquent potentiellement la « sélection implicite » d'un système normatif de gouvernement local, à savoir le modèle clientéliste (principalement dans les pays du Sud de l'Europe), celui de l'État providence (principalement dans les pays du Nord de l'Europe) ou celui du développement économique ${ }^{153}$. À ce titre, la construction de l'IAL devrait conduire à une exploration en profondeur des causes de l'autonomie locale pour mieux comprendre les trajectoires nationales. C'est finalement de sa capacité explicative que dépend la qualité de cet instrument comparatif.

Enfin, les scores des autres études empiriques ayant également évalué l'autonomie locale et la décentralisation comparativement, mais différemment, devraient être comparés avec les présents résultats, afin d'évaluer la validité de ces derniers et en améliorer la mesure. De même, il serait intéressant de se poser la question de l'applicabilité de l'IAL au-delà du contexte strictement européen.

\section{Remerciements}

Un grand merci aux Professeur.e.s S. Kuhlmann, A. Ladner et K. HorberPapazian pour leurs conseils avisés lors des différentes phases d'élaboration du présent article.

152. FLEURKE F., WILLEMSE R., op. cit., 2004.

153. GOLDSMITH M., «Local Autonomy: Theory and Practice », in KING D.S., PIERRE J. Challenges to Local Government, London, SAGE Publications, 1990, p. 15-36 ; PIERRE J., op. cit., 1990. 


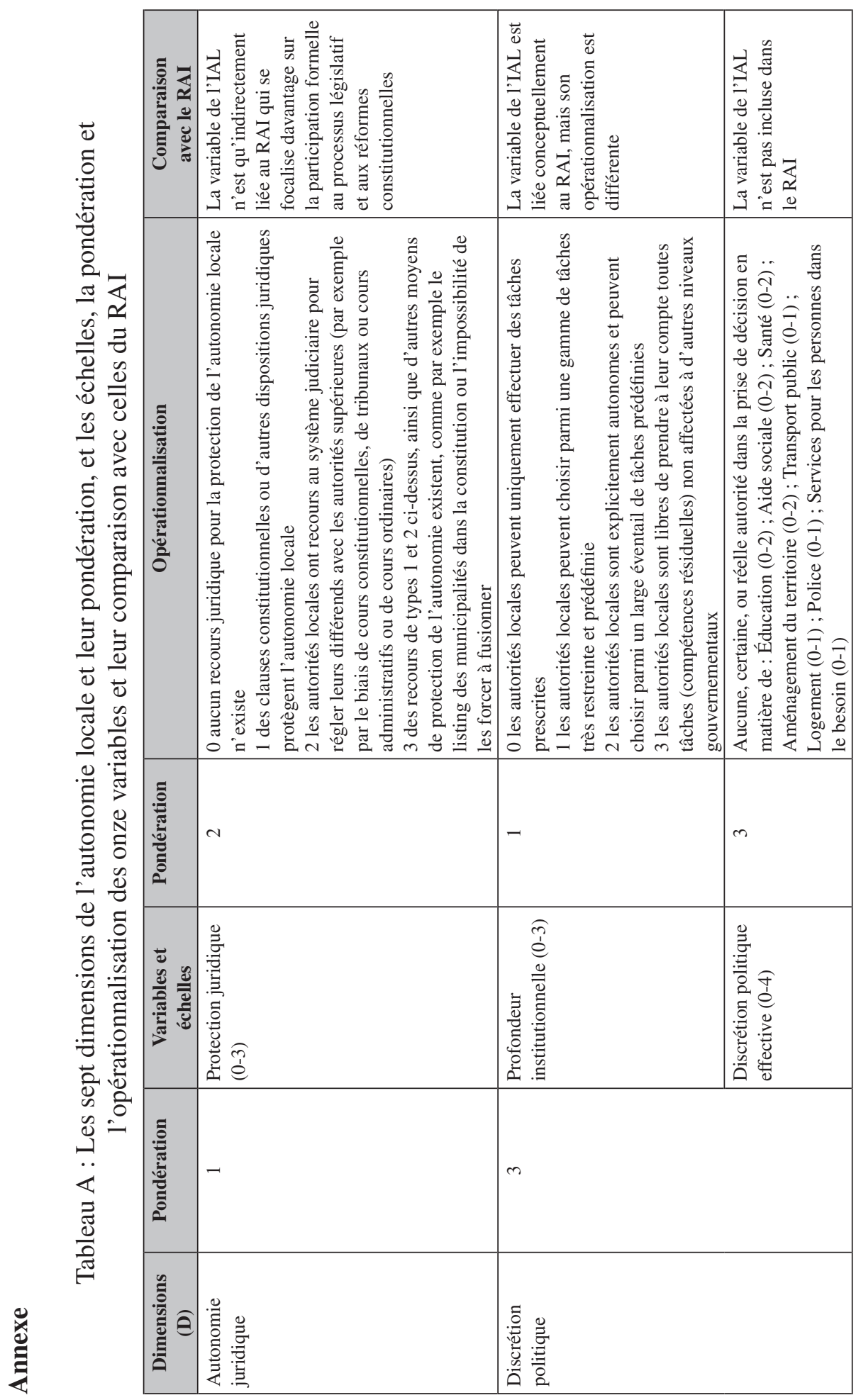




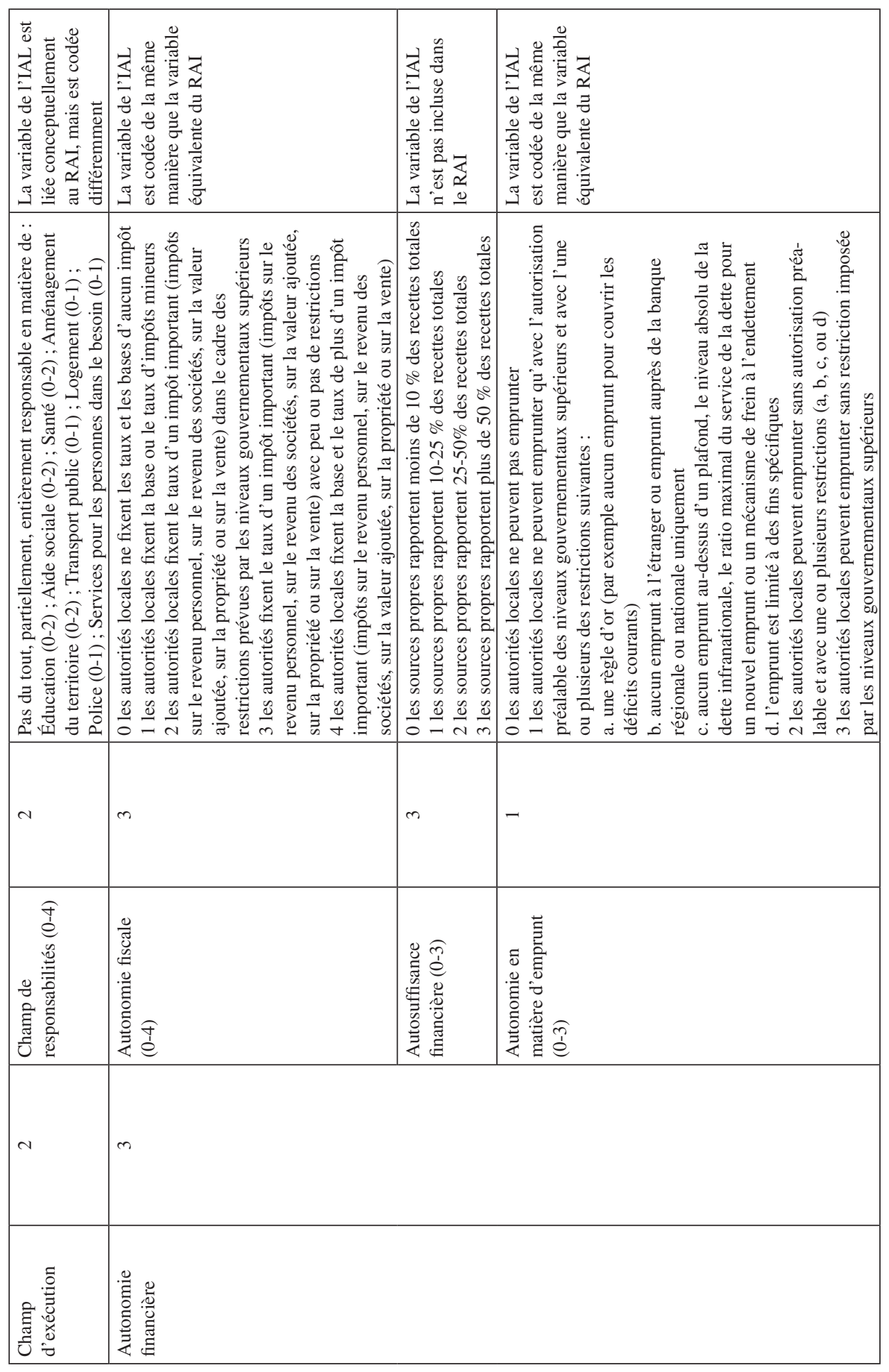




\begin{tabular}{|c|c|c|}
\hline 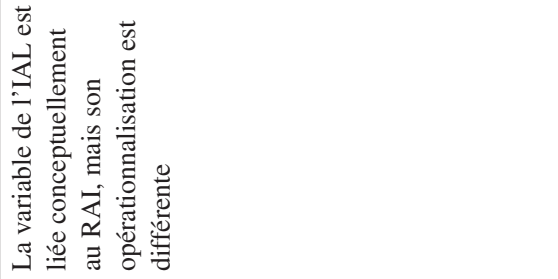 & 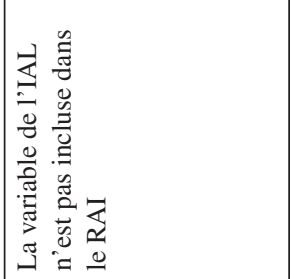 & 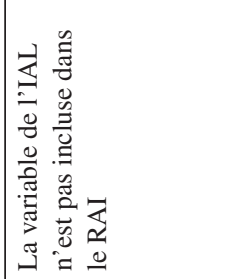 \\
\hline 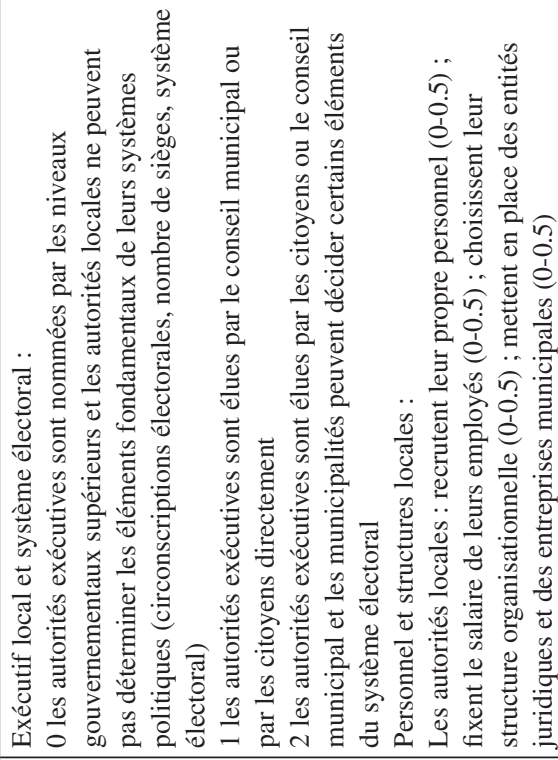 & 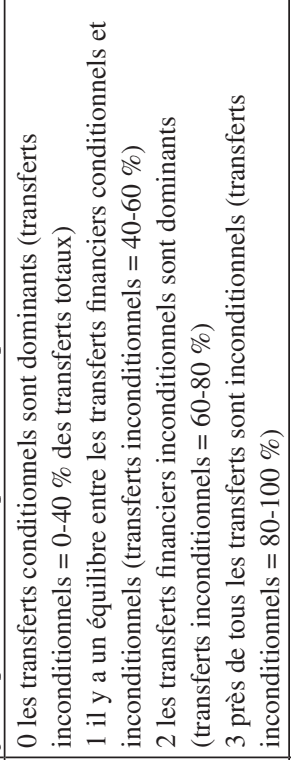 & 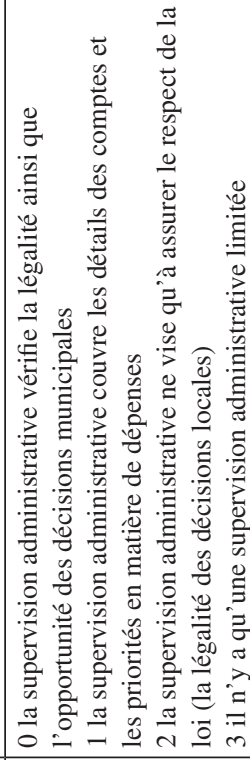 \\
\hline$m$ & - & - \\
\hline 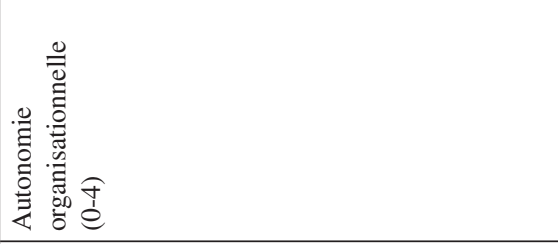 & 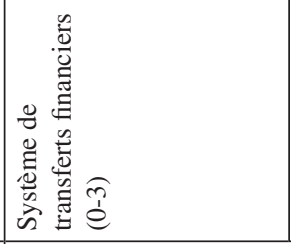 & 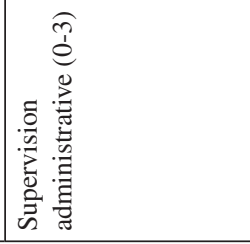 \\
\hline$N$ & - & \\
\hline 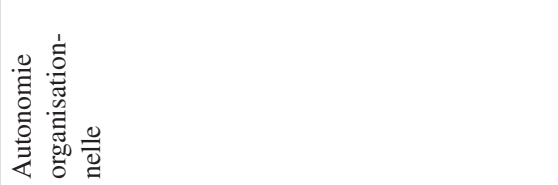 & 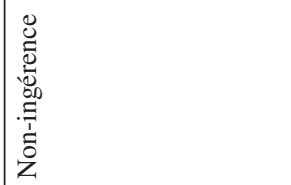 & \\
\hline
\end{tabular}




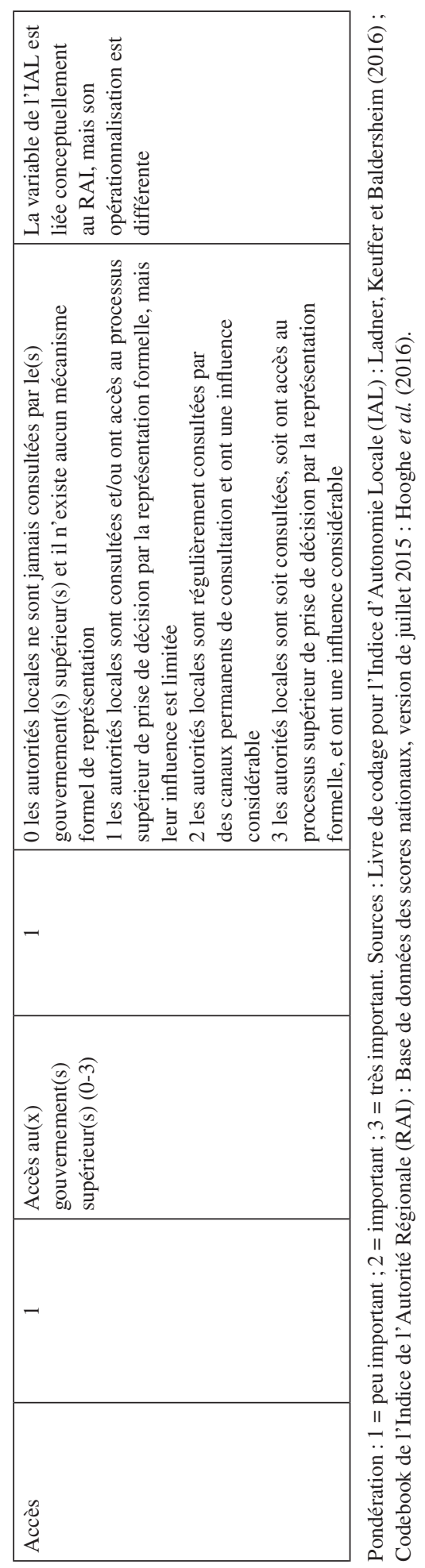




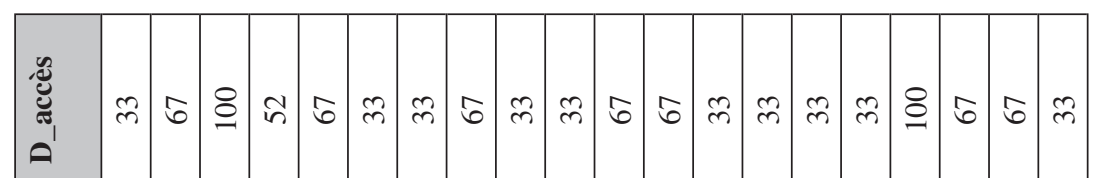

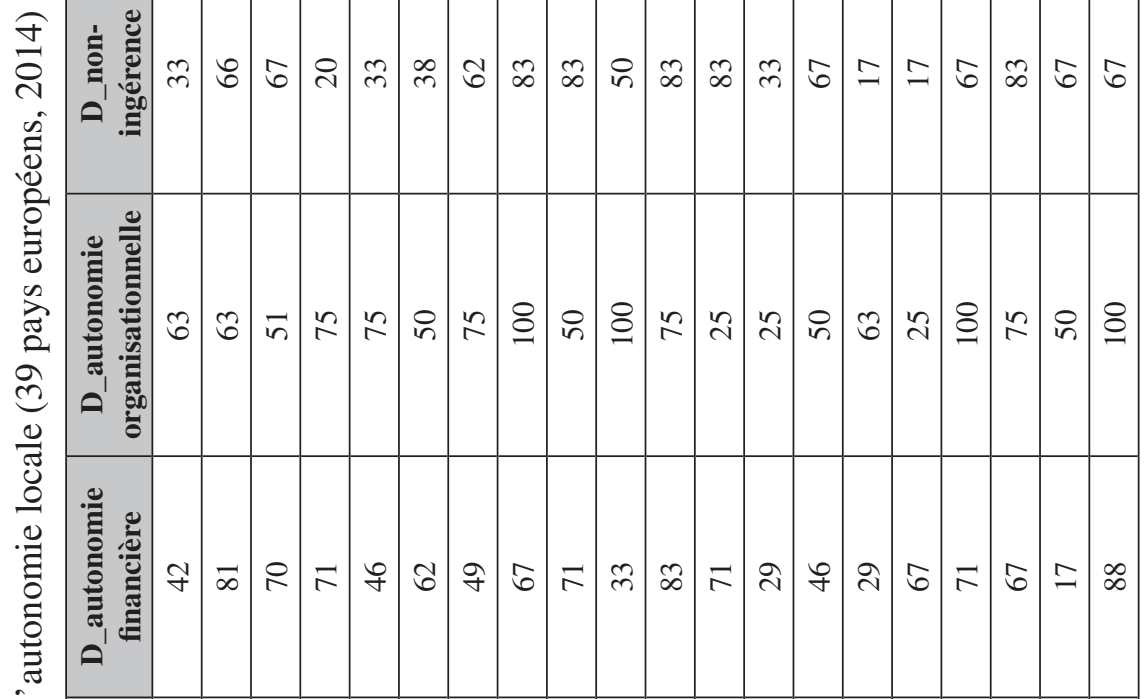

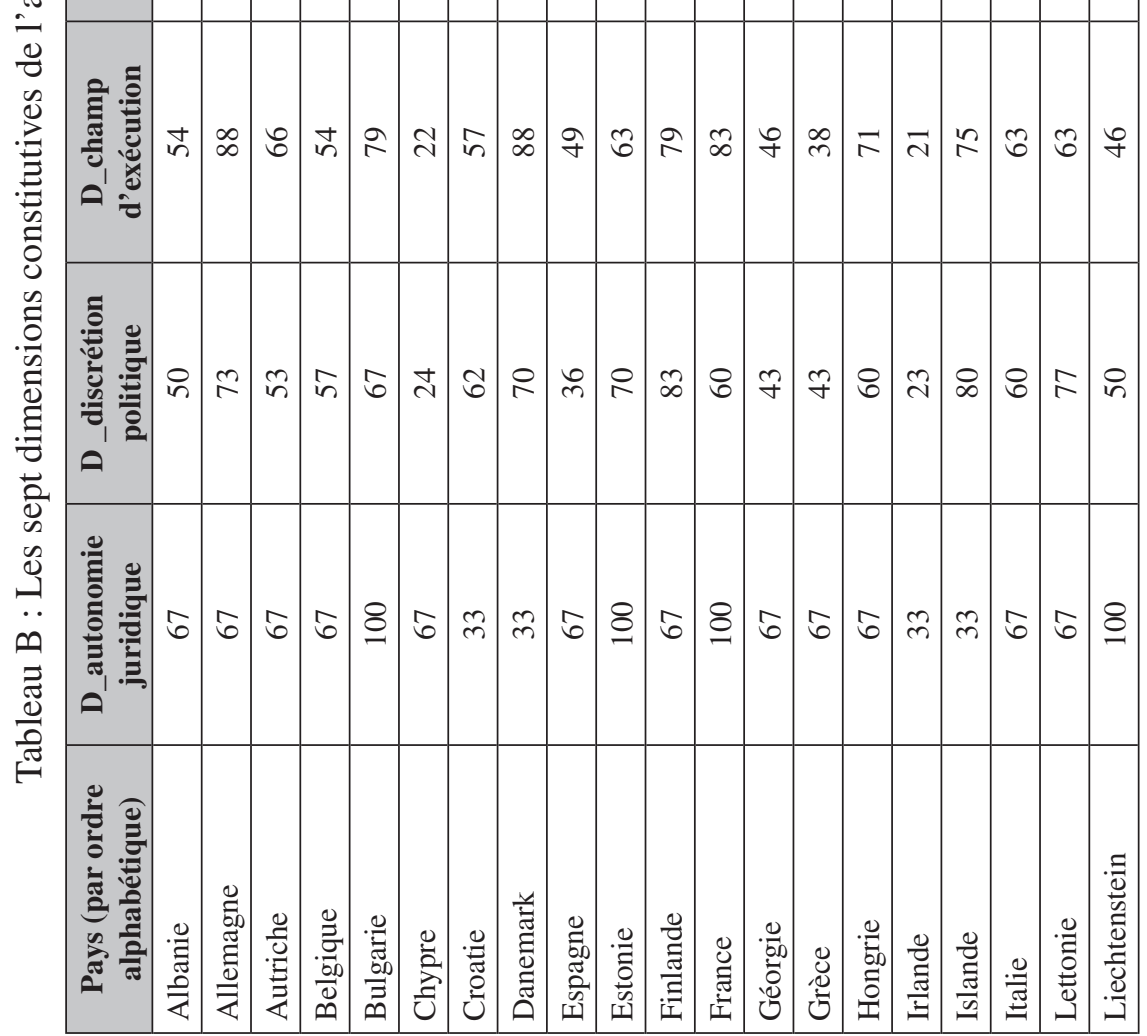




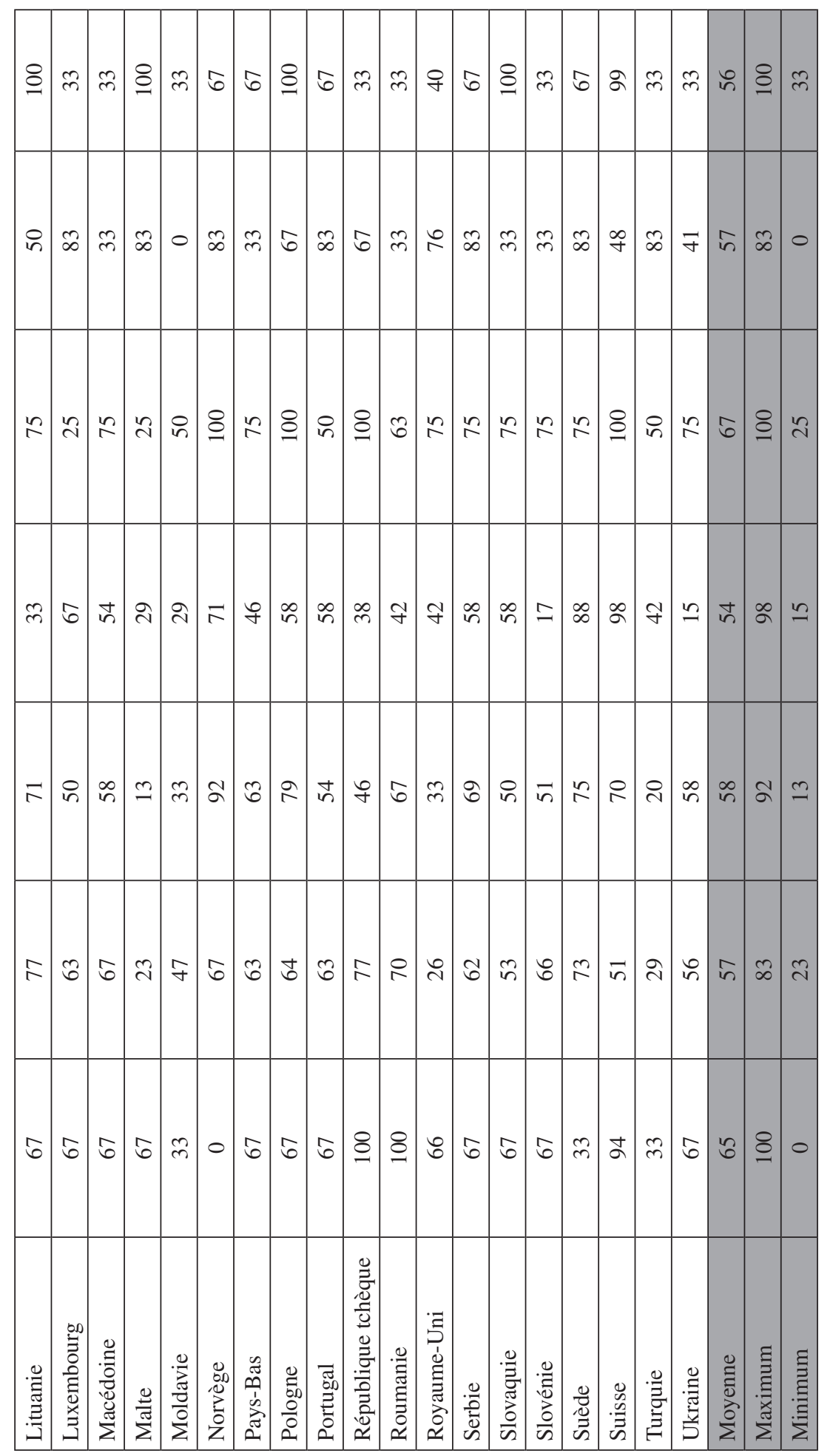




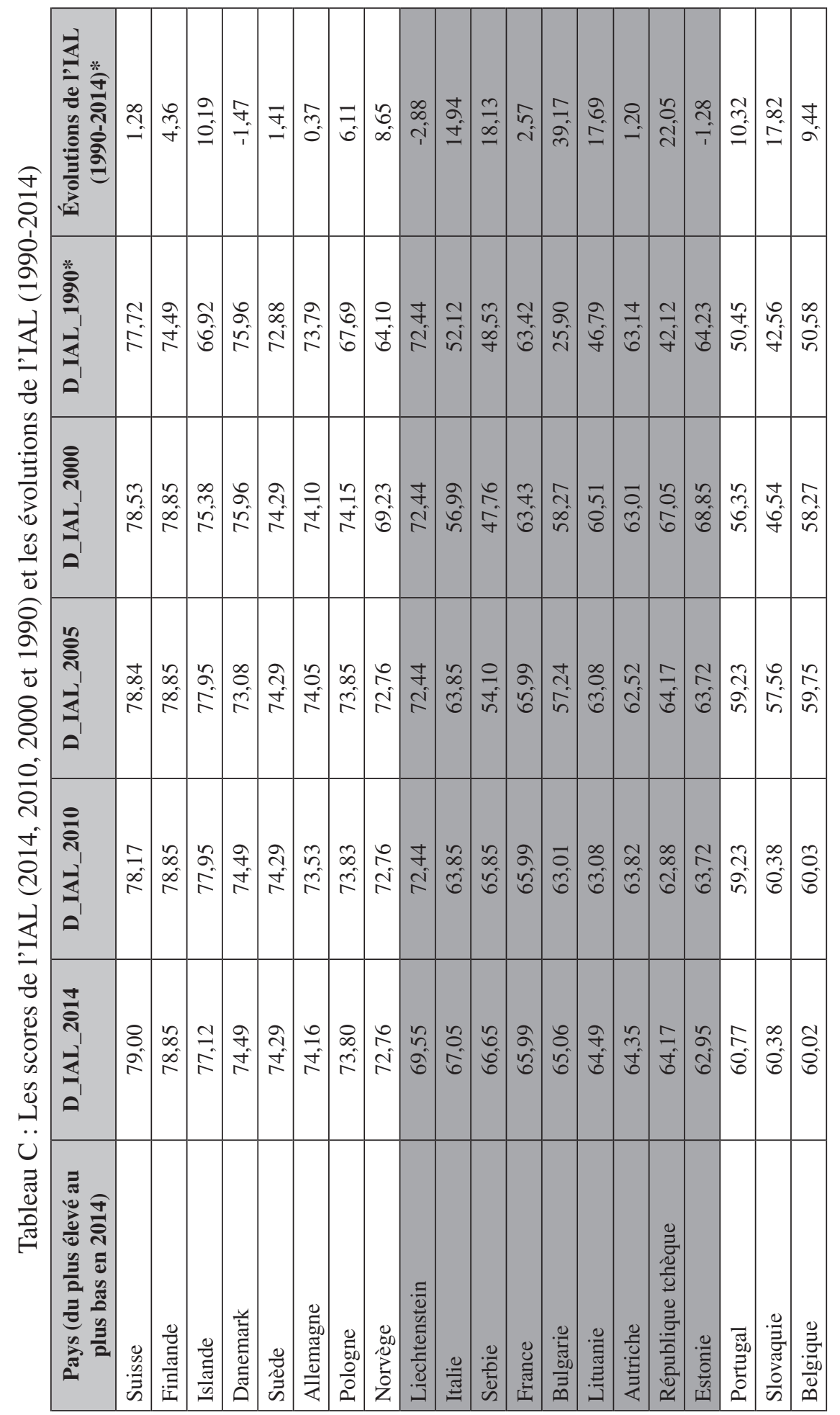




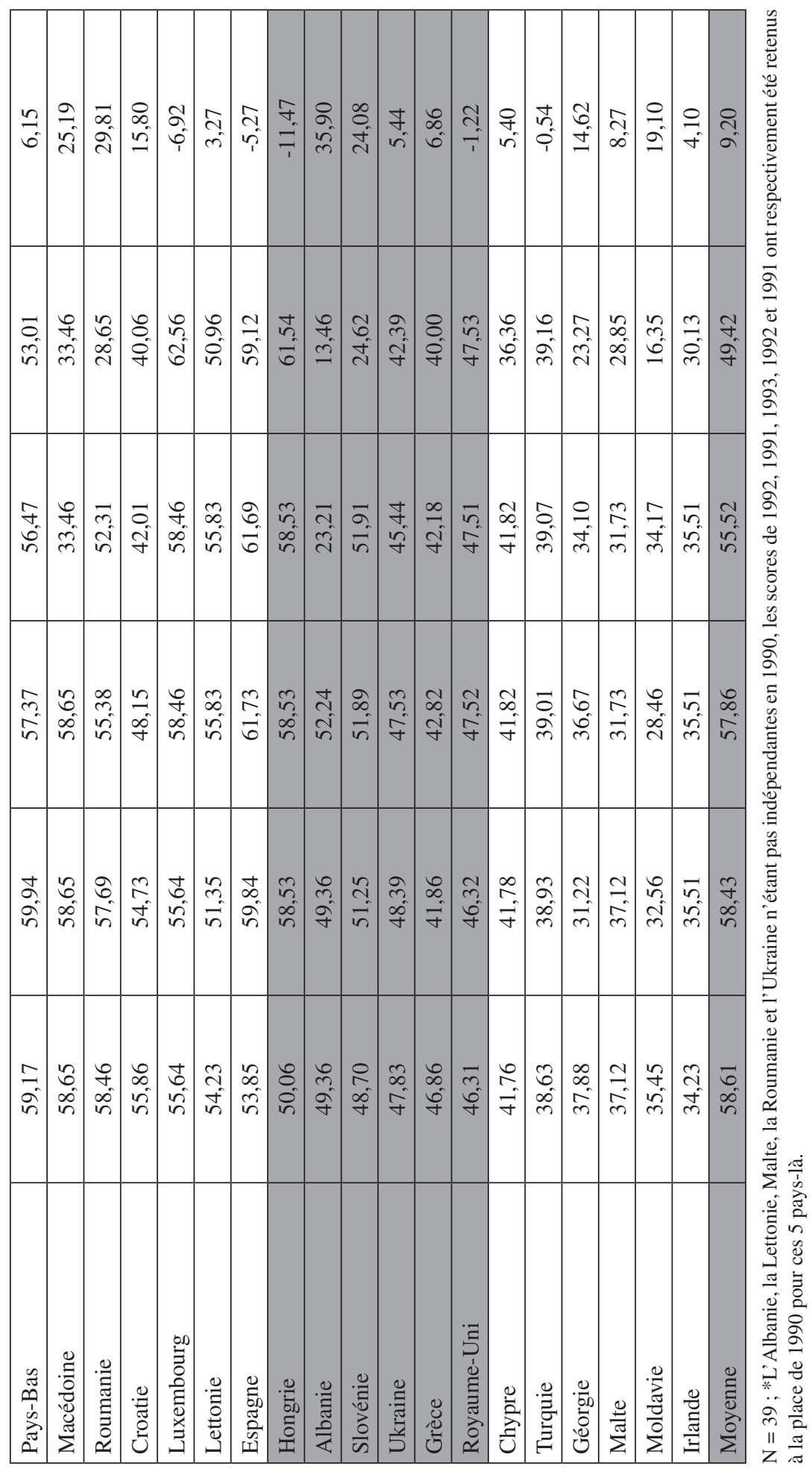

